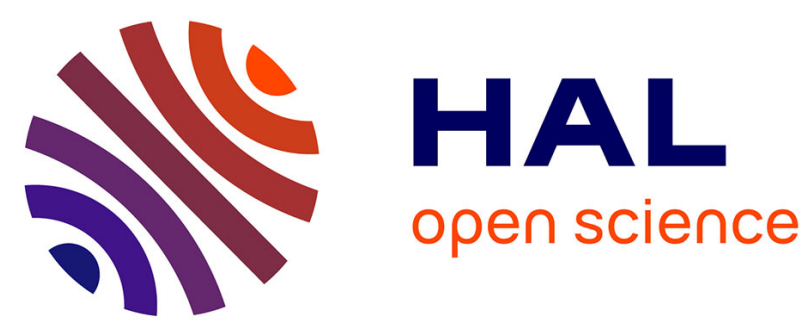

\title{
Cost-effectiveness assessment of three components of the bovine tuberculosis surveillance system by intradermal tuberculin testing in French cattle farms by a scenario tree approach
}

\author{
V. Poirier, J. Rivière, A. Bouveret, S. Gardon, Barbara Dufour
}

\section{To cite this version:}

V. Poirier, J. Rivière, A. Bouveret, S. Gardon, Barbara Dufour. Cost-effectiveness assessment of three components of the bovine tuberculosis surveillance system by intradermal tuberculin testing in French cattle farms by a scenario tree approach. Preventive Veterinary Medicine, 2019, 166, pp.93 - 109. 10.1016/j.prevetmed.2019.03.004 . hal-03485817

\section{HAL Id: hal-03485817 https://hal.science/hal-03485817}

Submitted on 20 Dec 2021

HAL is a multi-disciplinary open access archive for the deposit and dissemination of scientific research documents, whether they are published or not. The documents may come from teaching and research institutions in France or abroad, or from public or private research centers.
L'archive ouverte pluridisciplinaire HAL, est destinée au dépôt et à la diffusion de documents scientifiques de niveau recherche, publiés ou non, émanant des établissements d'enseignement et de recherche français ou étrangers, des laboratoires publics ou privés.

\section{(c) (1) $\$$}

Distributed under a Creative Commons Attribution - NonCommerciall 4.0 International 
1 Cost-effectiveness assessment of three components of the bovine tuberculosis surveillance system by intradermal tuberculin testing in French cattle farms by a scenario tree approach

3 V. Poirier ${ }^{1}$, J. Rivière ${ }^{1}$, A. Bouveret ${ }^{2}$, S. Gardon ${ }^{3}$, and B. Dufour ${ }^{1}$.

$4 \quad{ }^{1}$ USC EPIMAI, Anses, Ecole Nationale Vétérinaire d'Alfort, F-94700, Maisons-Alfort, France

$5 \quad{ }^{2}$ Animal Epidemiology Working Group of the military health service, F-75007, Paris, France Marcy l'Etoile, France.

Corresponding author: Valentine Poirier, USC EPIMAI, Anses, Ecole Nationale Vétérinaire d'Alfort, 7 avenue du Général de Gaulle, F-94700, Maisons-Alfort, France; Email: valentine.poirier@ vetalfort.fr ; Telephone number: +33(0)1 43967008

Declarations of interest: none

In most officially bovine tuberculosis (bTB)-free countries, bTB has not been fully eradicated. Costly and time-consuming surveillance and control measures are therefore still in place to control this infection. An officially bTB-free status, both at the national and at the herd level, influences whether and when animals can be sold. Thus, this infection is still an economic issue, justifying measures towards its eradication. An evaluation of the cost-effectiveness of such measures would be highly

ADL: accredited departmental laboratory for bTB analyses

BDNI: National Data Base of Identification (listing of every cattle living in France)

bTB: bovine tuberculosis

CEA: cost effectiveness analyse

DNA: deoxyribonucleic acid

ICCT: intradermal cervical comparative tuberculin test

ICT: intradermal cervical tuberculin test

IFN: $\gamma$-Interferon test

NLR: national laboratory of reference for bovine tuberculosis (LNR Anses Maisons Alfort, France)

SICT: single intradermal cervical tuberculin test 
useful, especially to optimise the costs of control measures and their adaptation to a local epidemiological context. We evaluated the cost-effectiveness of three mandatory surveillance protocols currently used in France by herd type (type of production, size, and turnover of the herd) under French field conditions. The first protocol ("strict") implies the direct slaughter and post-mortem analyses of any intradermal cervical tuberculin test (ICT) reactor, and negative results to a second intradermal cervical comparative tuberculin test (ICCT) to regain bTB-free status of the herd. In the second protocol ("compliant quick-path") bTB-free status can be regain if post-mortem analyses of reactors to the first ICT are negative. In the third protocol ("compliant slow-path"), ICCT-reactive animals are tested using the interferon gamma assay; the results of this test influence the path of further investigation. We built scenario trees for each of these protocols at the animal level. They allowed us to estimate herd sensitivity and the total cost of each protocol by herd type. The protocols could be ordered by decreasing herd sensitivity and cost, regardless of the herd type, as follows: strict protocol, compliant quick-path protocol, and compliant slow-path protocol. We calculated a costeffectiveness index to evaluate the cost-effectiveness of each protocol. The strict protocol was never the most cost-effective, regardless of herd type, due to higher costs relative to the other protocols, despite better herd sensitivity. We found the compliant quick path to be the most cost-effective protocol for big beef, big dairy, and mixed herds. The compliant slow path was the most cost-effective for small-scale beef and dairy herds. All differences were significant. This comparison of the costeffectiveness of the protocols by herd type could help authorities to choose the most suitable protocol in the investigation of suspected cases, depending on the herd type, but could be improved by accounting for important sociological data, such as the acceptability of the protocols.

Keywords: scenario tree, bovine tuberculosis, surveillance, sensitivity, cost, cost-effectiveness.

\section{INTRODUCTION}

Bovine tuberculosis (bTB) is a chronic disease mainly caused by Mycobacterium bovis. This infection can be zoonotic (Müller et al., 2013), and in developed countries, bTB is mainly an economic issue. 
Indeed, in developed countries, zoonotic tuberculosis in humans is insignificant (Thoen et al., 2006), whereas bTB results in major economic losses to farmers due to sales restrictions and to governments due to surveillance expenses and compensations for slaughtered cattle. In France, bTB has not been fully eradicated, despite the bTB-free status given by the European Union in 2000 (decision 2001/26/CE of 27 December 2000) and, thus, surveillance and control measures are still in place. These measures require significant economic and human resources, which are limited in the current economical context. France is aiming to eradicate bTB, as several other European countries, requiring improved cost-effectiveness of bTB surveillance and control systems.

Improvement of the surveillance system first requires an evaluation of those already in place. The actual French bTB surveillance system relies on the systematic inspection of carcasses in slaughterhouses, screening of bovine livestock (programmed screening in farms and purchase testing), and epidemiological investigation in farms linked to breakdowns. We present part of the current evaluation program of the French bTB surveillance system. It focuses on one of the three components: programmed screening in farms, putting aside purchase testing, slaughterhouse surveillance and investigation in the farms epidemiologically linked to breakdowns. We developed scenario-tree models for each subcomponent (or protocol) of programmed screening. These trees allowed us to assess the effectiveness of each of the subcomponents and their cost, depending on the type of herd (type of production, size, and herd turnover). The aim was to produce an evaluation that could help decisions-makers to choose the most cost-effective investigatory protocol, depending on the herd type considered.

\section{MATERIALS AND METHODS}

\subsection{French bTB surveillance system on farms}

In France, veterinarians regularly perform programmed screening of cattle herds. They test every animal older than 24 months (adults) with an intradermal cervical tuberculin test (ICT). This ICT can be a single intradermal cervical tuberculin test (SICT), which is an intradermal injection of bovine tuberculin in the middle of the neck, or an intradermal cervical comparative tuberculin test (ICCT), 
which consists in injecting bovine tuberculin and avian tuberculin in two different areas of the neck and is interpreted by comparison of the skin thickening at this two points 72 hours later. Here, we investigated the protocols using the ICCT as a screening test because it is already used as a routine test in several countries, such as the Republic of Ireland and the United-Kingdom (EFSA, 2012), and France is currently replacing the SICT with the ICCT. A herd is considered to have a suspected bTB infection if at least one animal is non-negative (e.g. positive or doubtful) for the ICCT. The farmer of a suspect herd cannot sell their animals and the authorities must carry out further investigations.

Veterinary authorities use mainly three investigation protocols. For all, the final decision concerning the bTB status of a suspect herd (confirmation as a breakdown or regaining bTB-free status) depends on the results of laboratory tests described below. Suspect animals are slaughtered, and a thorough inspection of the carcass is conducted. Three lymph nodes are systematically sampled (retropharyngeal, tracheobronchial, and mediastinal) and any suspect lesion and their corresponding lymph node identified. Samples are then sent to an accredited departmental laboratory (ADL) for real time PCR and bacteriological culture(s), which are systematically performed by the ADL; and for histological analysis on lesions if presents. If positive or doubtful results are obtained, samples are sent to the national laboratory of reference for bTB (NLR) were the diagnosis is confirmed by real time PCR, genotyping (on PCR products to confirm diagnosis of doubtful results and to differentiate mycobacterial species), and/or mycobacterial culture.

The path leading to reclassify a herd as bTB-free depends on the protocol used. When it seems likely that the farm is truly bTB infected (at least one positive ICCT, former breakdown, etc.), reclassification requires re-evaluation of the herd with a second ICCT 42 days later and the slaughter of any reactive animals for laboratory analyses: this constitutes the "strict" protocol. During this protocol, no animal can be sold. Otherwise, there are two "compliant" protocols. The "quick-path" permits direct (and therefore faster) reclassification of the herd without re-evaluation by ICCT if negative results are obtained following slaughter of the first reactive animals. The "slow-path" avoids the direct slaughter of reactive animals: blood samples from reactive animals are taken three days after ICCT injection (when the veterinarian come back in the farm for ICCT reading) and sent to the ADL for IFN testing. The IFN assay is performed using Bovine and Avian Lelystad PPD (Bovigam®; 
ICCT non-negative at the first screening (ICCT1) are slaughtered for diagnosis. Meanwhile, selling any animal from the herd is prohibited. If no IFN bTB-positive animal is detected, animals that were non-negative for the first ICCT (ICCT1) are tested by a new ICCT (ICCT2) 42 days after the first one and the selling of ICCT1-negative animals is authorized (only to fattening farms when at least one non-conclusive IFN test was found and without other conditions). If all tested animals are negative for the second ICCT, the herd regains its bTB-free status. Otherwise, reactive animals are slaughtered for diagnosis. The use of serial IFN testing is not recognized by the European Union as a discriminant test to confirm or infirm bTB status of an herd. Compliant slow-path protocol and compliant quick-path protocol are consistent with that and unique in France to relieve the movements constraints on the national level.

Scenario tree modelling is frequently used to prove freedom from disease according to a fixed minimum prevalence that the surveillance system should be able to detect (Martin et al., 2007; Hadorn et al., 2009). This method consists of representing each protocol as an event tree that describes each possible pathway from infection of the population to its detection by the protocol studied. Nodes composing the scenario tree separate the studied population into groups of animals with the same probability of being infected and the infection detected. A specific probability is associated with each node outcome. The probability of occurrence of each pathway of the tree is obtained by multiplying the probabilities along the corresponding limb. We used this method to assess the herd sensitivity and cost of the three bTB surveillance protocols using a stochastic approach for the types of farms already described (type of production, size and herd turnover). We fixed the probability of infection alternatively to 0 or 1 , depending on the variable to estimate (herd sensitivity, herd specificity or total cost of the considered protocol). We defined the protocol herd sensitivity as the probability that at least one infected animal of an infected farm would be detected by the protocol. 
129 Figures 1 to 3 illustrate the nodes and input parameters of the trees for each of the surveillance

130 protocols for cattle farms. Most of the input parameters are described by distributions, to account for

131 the variability and uncertainty of the estimates. We used common distribution laws (normal, PERT, or

132 uniform) and normal asymmetric distribution laws (Sicard, 2013) (Appendix 1). All distribution laws

133 representing the probabilities of occurrence were restrained between 0 and 1 . The parameters of the

134 laws were estimated based on French databases and the scientific literature. The SIGAL database

135 (centralized national database of the ministry of food and agriculture) provided data concerning the

136 surveillance and control interventions (screening results and laboratory analyses) in 2016. The national

137 database of cattle identification (BDNI) provided data concerning the distribution of production type,

138 herd size, distribution of age within the herds, and animal movements (sales) in 2016. The categories

139 of farms listed below were defined by the Omar project (Perrin et al., 2012) and were available in the

140 BDNI. We used herd type data from a French department in which each herd type (production type,

141 size, and turnover category) was present and in which the size and proportion of adult cattle were the

142 closest to the National French average for each herd type (data of 1 January 2016 from Cantal,

143 extracted from the BDNI).

144 For each herd type, the mean herd sensitivity and cost of a screening protocol and their 95\%

145 confidence interval (CI) were estimated for a one-year period (bTB is a chronic disease and screening

146 in farms occurs every year) from 10,000 simulated values. The scenario trees were implemented in

147 RStudio (version 1.1.383.0).

$148 \quad$ 2.2.1. Herd type

149 The aim of this study was to evaluate bTB surveillance on farms for each of the twelve herd types

150 defined by:

151 - Their type of production: dairy, beef (except for strictly fattening farms, which are exempt

152 from the program), and mixed farms (with both dairy and beef activities). Each type of

153 production has a different age distribution; the proportion of animals tested will thus be

154 different for each production type, influencing the probability of detecting a bTB infection in

155 the herd. 
- Herd size: "big" and "small". A "small" herd has a number of adults inferior to the national average for this type of production (74 for dairy farms, 66 for beef farms, and 124 for mixed number of animals confirmed to be infected in infected herds is generally between one and three

180 (Poirier, 2017). In 2017, Bouveret (Bouveret, 2017) reported a within-herd prevalence rate between farms); otherwise, it is a "big" herd. Herd size mainly influences the cost and herd sensitivity of surveillance through the number of screened animals and the specificity of protocols through the probability of false-positive results.

- Herd turnover: we used the French limit of $40 \%$ to distinguish low from high turnover. Herd turnover was defined as the number of cattle introduced into the herd (excluding birth) within the year, divided by the mean number of cattle during the year. Herd turnover can have an impact on the cost, as farms with a high turnover may suffer more indirect economic losses due to bans on the selling of animals.

2.2.2. Age of the animals

Young animals (younger than 24 months) are not screened in the bTB surveillance system. The age of the animal is thus a factor that influences its probability to be tested and thus detected. We used normal distribution laws to describe the proportion of adults in each type of herd. The parameters of such normal distributions were fitted to the actual distribution data from the BDNI (fitdistrib R function) (Table 1).

2.2.3.bTB prevalence at herd and individual levels

The probability of infection at the herd level was fixed at one to estimate the herd sensitivity and costs of each protocol for infected herds and at zero to estimate the costs for uninfected herds.

Within-herd prevalence has been estimated only once in France, in 2005, in a beef farm herd of Côted'Or. In this unpublished study, only two animals were found to be infected, corresponding to a within-herd prevalence of $0.94 \%$. However, this study used conventional PCR on blended organs instead of the real-time PCR technique used today, which has better sensitivity (Mackay, 2004). The $0.8 \%$ and $3.1 \%$ in French breakdowns between 1988 and 2008. Based on these results, we choose to 
describe the probability for an animal of an infected herd to be infected by a PERT distribution law: PERT $(\min =0.008$, mode $=0.0094, \max =0.031)$.

\subsubsection{Proportion of animals tested}

Screening generally misses a few adults, primarily for technical reasons (for example, difficult tocontain animals). Based on the SIGAL database, we estimated that between $95 \%$ and $99 \%$ of adult animals are tested. We thus used a uniform law between 0.95 and 0.99 to describe this parameter. The data did not allow differentiation by herd type.

In the strict protocol, only a sampling of animals in the suspect herd is generally tested a second time with ICCT. Analysis of the SIGAL database showed a minimum of one adult to be tested a second time and a maximum of $100 \%$. Limited data were available in this database (207 farms) and we detected no significant difference in the mean number of animals tested a second time between herd types. Nevertheless, all animals of most (55\%) small herds appeared to be tested a second time with ICCT, whereas the average number of animals tested a second time in big herds was 53 . The proportion of adults tested a second time with ICCT in the strict protocol was finally described by a PERT distribution, for which the parameters were chosen by herd size according to the following data: PERT $(\min =1 /$ herd size, mode $=1, \max =1)$ for small herds and PERT $($ min $=1 /$ herd size, mode $=$ $53 /$ herd size, $\max =1$ ) for big herds. The representation of herd size in the models is described in section 2.2.8.

\subsubsection{Ante mortem tests results}

\subsubsection{ICCT}

Estimations of sensitivity and specificity vary widely in published studies. We chose to combine all estimations into normal asymmetric distribution laws to represent ICCT and its uncertainty (Table 2). In the strict protocol, ICCT1-negative animals are tested again by ICCT six weeks later. We found no information concerning the characteristics of a second ICCT (ICCT2) after a first negative ICCT result. We assumed that the result for the second ICCT has the same characteristics as the first ICCT, because animals screened by ICCT at year $n$ are mainly animals already negative to the screening with an ICCT at year $n-1$. 
In the slow-path compliant protocol, ICCT1 non-negative animals are tested again by ICCT six weeks

after the ICCT1. Parameters used to represent the sensitivity and specificity of ICCT2 conditional to a first non-negative result to ICCT1 are presented in Table 2.

\subsubsection{IFN gamma}

The characteristics of IFN testing have already been studied under French field conditions (Praud and Dufour, 2016). This study was used to set the parameters of normal distribution laws that describe the sensitivity and specificity of IFN testing presented in Table 3.

\subsubsection{Post mortem tests results}

The diagnostic slaughter test results are represented separately in Figure 4, as the path is the same for each of the three screening protocols studied. The sensitivity and specificity of bTB tests (PCR, bacteriology and histology) were previously estimated depending on the presence of bTB-like lesions (Courcoul and Boschiroli, 2013). As the CIs of these estimations were asymmetric, we used normal asymmetric distribution laws for the parameters of the scenario tree (Table 4). We calculated the proportion of animals with detectable lesions, depending on the infectious status of the animal, using French slaughter data between 2008 and 2012, described by Courcoul et al., 2014 (Appendix 2).

We hypothesised that the histology was independent of PCR and bacteriology, based on the modelling results of Courcoul et al. (2014), but accounted for the inter-dependence of PCR with bacteriology.

We represented the covariance of the sensitivity and specificity between PCR and bacteriology, depending on the presence of detectable lesions by PERT distribution laws. The modes of the PERT distributions were chosen based on the results of Courcoul et al. (40\% of the maximal covariance when detectable lesions are present and a covariance equal to zero when there are no detectable lesions). We defined the minimums and maximums of these laws as the minimum and maximum value possible for the covariance between the two tests used in parallel (Gardner et al., 2000; Appendix 3).

232 We assumed that tests (PCR, bacteriology, and genotyping) performed by the NRL are perfectly 233 sensitive and specific (personal communication, M-L. Boschiroli, NRL director). 
In strict and compliant slow-path protocols, the path followed by an animal depends on the test results of the entire herd (Figures 2 and 3). Indeed, the probability for ICCT-negative animals to be tested 42 days later in the strict protocol (Figure 2) is the probability that at least one animal in the herd is ICCT non-negative at the first screening and that laboratory bTB analyses performed on slaughtered animals are negative. The probability for ICCT1 non-negative and IFN non-positive animals to be tested a second time with ICCT2 in the compliant slow-path protocol (Figure 3) is the probability that no IFNpositive animal is found in the herd. Herd size was essential for calculating these probabilities. We thus described herd size (number of animals present in the herd) by herd type with normal distribution laws fitted to the distribution of BDNI data (Table 5).

Costs of surveillance protocols can be direct (screening tests, organisation of the screening campaigns, laboratory analyses, etc.) or indirect (losses due to the ban of selling animals or unpasteurised milk). In France, the State and farmers share these costs. Farmers must pay for programmed screening and suffer indirect losses, whereas the State pays for all tests applied during the investigation of suspected cases and compensates farmers for slaughtered animals. All following prices and costs are tax-free.

\section{Direct costs}

Each year, each department sets the price for bTB screening tests for the next screening campaign by a written agreement between veterinarians and farmers. We collected 40 such agreements for 2015 to 2017. We corrected the prices based on the inflation rate between the agreement year and 2017 $(0.2 \%)$, if necessary. The mean, maximum, and minimum of these data were used for the parameters of the PERT distribution laws used to describe the cost of a screening visit in euros per visit (considering that two visits are needed to perform the tuberculin injection and the reading of the ICT results) and the cost of an ICCT injection in euros per animal. The cost of an ICCT injection was therefore described as PERT $(\min =5.01 €$, mode $=7.36 €, \max =9.94 €)$, and the cost of the visit as PERT $(\min =30.06 €$, mode $=53.58 €, \max =70.90 €)$. The State sets the prices of ante mortem tests for investigations for suspected cases. In 2017 , they were $28.36 €$ for the veterinarian visit (injection and reading), $7.09 €$ per animal for ICCT2, and $2.84 €$ per animal for blood sampling for the INF test. 
In France, the price of post mortem analyses is different for each laboratory. Thus, we conducted an online survey among the 40 French ADLs in April 2018 with the help of the French association of the directors and executives of veterinary laboratories (ADILVA). We used the results of this survey to estimate the price of each analysis and sample transport fees (Table 6). Based on the SIGAL database, we estimated the mean number of samples sent for PCR by an ADL for one animal, depending on the presence of detectable lesions (3.59 for animals with lesions and 3.77 for animals without lesions) and the mean number of samples sent for histology by an ADL (1.9 samples for animals with lesions only). These data and the probability of occurrence of each laboratory analysis allowed us to calculate the mean cost of the diagnostic slaughter for an animal, depending on its infectious status. The State compensates farmers 2,200€ for the slaughter of an adult animal if it is registered in the herd book and 1,900 € otherwise (ministerial decree of 17 June 2009); the meat value of the animal is deducted from this compensation. We represented the meat value by normal distribution laws designed to fit the best SIGAL data on the meat value of animals slaughtered during bTB investigations of suspected cases in 2016. The meat value of adults was therefore represented by normal distribution laws: Normal $($ mean $=972.15 €, S D=314.89 €)$ for beef farms, Normal $($ mean $=633.04 €, S D=$ $284.32 €)$ for dairy farms, and Normal $($ mean $=628.07 €, S D=434.22 €)$ for mixed farms. We estimated the percentage of cattle registered into the herd book (herd book inscription rate) for each production type to be $20 \%$ for dairy cows, $10 \%$ for beef cows, and $15 \%$ for cattle of mixed farms (source: http://www.racesdefrance.fr). We finally estimated the final compensation cost by the State with formula (1).

(1) compensation $(€)=(1$-herd book inscription rate $) * 1900+$ herd book inscription rate $* 2200-$ meat value

We associated a weighted direct cost to each limb of the tree, equal to the corresponding direct cost multiplied by the probability of occurrence of the limb. Then, we calculated the mean direct cost for each protocol by herd type, by adding all weighted costs of the paths corresponding to the considered

287 herd type. 
We represented the indirect costs due to a sales ban using a French evaluation of the daily-care costs (feeding, veterinary fees, supplementary work for the farmer, etc.) for each type of animal (age, sex, and production type) (Buczinski and Reuillon, 2015)). The values of these estimations were collected to estimate daily-care costs for females younger or older than 24 months, males younger or older than six months, and the total indirect costs of dairy calves of dairy, beef, and mixed farms, as described in Appendix 4. We used the estimations from this study to describe the cost of care $\left(\mathrm{C}_{\mathrm{t}, \mathrm{X}}\right)$ by animal ( $\left.\mathrm{t}\right)$ and production $(\mathrm{X})$ type (Table 7). The indirect cost of dairy calves is not daily but covers the entire period of the sales ban, because it accounts for the fact that farmer will be forced to keep the animal a certain amount of time before it can be sold as a fat dairy calf.

This indirect cost is only incurred for animals which would have been sold but were not because of a sales ban. Thus, we extracted the number of each type of animal sold each month of 2016 for each type of herd from the BDNI database. We estimated the means and standard deviation of the normal distribution law that best fit these data using the "fitdistrib" R function for each type of herd. The resulting means and standard deviations were used as the parameters of the normal distribution laws (restrained between 0 and 200 (the maximum number of animals sold based on the data)) represented by $\mathrm{n}_{\mathrm{m}, \mathrm{t}, \mathrm{X}}$, the number of each type of animal $(\mathrm{t})$ sold each month $(\mathrm{m})$ in each type of herd $(\mathrm{X})$

(Appendix 5). From SIGAL, we determined the proportion of suspicious cases for each month (m) of $2016\left(\mathrm{APMS}_{\mathrm{m}}\right)$. From these two sets of values and the number of days in each month $\left(\mathrm{J}_{\mathrm{m}}\right)$, we calculated, for each model iteration and each herd type, C_tot $x$, the mean indirect cost due to a sales ban for D days, in euros, for the herd type $\mathrm{X}$ with formula (2).

In formula (2), $C_{X}$ is the indirect cost for a herd type $X$, without considering dairy calves, calculated with formula (3), and $\mathrm{C}_{\mathrm{X} \text {,dairy calves }}$ is the indirect cost for a herd type $\mathrm{X}$ due to dairy calves, calculated with formula (4). 
In the compliant slow-path protocol, the blocking of all animals that should be sold depends on the results obtained at the IFN testing (2.1., Figure 3). We considered this particularity in our estimation of the indirect costs for the compliant slow-path protocol.

We calculated D, the mean duration of sales ban, for each protocol at each model iteration, by multiplying the delay between each protocol step (represented by the distribution laws presented in

Table 8) with their probabilities of occurrence in the scenario trees.

\subsubsection{Herd-level sensitivity}

For each protocol, we calculated the herd sensitivity for each type of herd. The prevalence of bTB in the concerned area was fixed at $100 \%$ to estimate the probability of detection of at least one infected animal in an infected herd. First, the model calculated the probability of occurrence of each path by multiplying the probabilities of occurrence for the corresponding limb. Then, for each protocol, it estimated the individual sensitivity for each type of herd $\left(\mathrm{Se}_{\mathrm{k}}\right)$ by adding the probability of occurrence of every path beginning with the nodes corresponding to the herd type $\mathrm{k}$ with a positive outcome. By construction, individual specificity was equal to $100 \%$. Finally, for each protocol, the herd sensitivity for each herd type (CSe $)$ was obtained by formula (5).

(5) $\mathrm{CSe}_{\mathrm{k}}=1-\left(1-\mathrm{Se}_{\mathrm{k}}\right)^{\text {herd size*intra-herd prevalence }}$

\subsubsection{Costs}

We calculated the mean direct and indirect costs for infected (bTB prevalence in the considered area fixed to $100 \%$ ) and uninfected herds (bTB prevalence in the considered area fixed at $0 \%$ ), by herd type, for each surveillance protocol, as described in section 2.2.9. The total cost calculated by herd type for each protocol corresponds to the sum of the mean direct and indirect costs.

\subsection{Analyses}

All analyses were performed using RStudio (version 1.1.383.0). The means were compared using Student's t test, corrected for multiple comparisons by the Bonferroni correction method with an accepted error risk $\alpha$ of $1 \%$. 
343 CEA was performed to assess the efficiency of the three studied surveillance protocols for each type of

344 herd. For each protocol, a cost/sensitivity index was calculated for each model iteration and each type

345 of herd. We therefore obtained the mean cost/sensitivity index and its $95 \%$ CI for each protocol from

34610,000 iterations for each type of herd.

348 We evaluated which model input had the most influence on outcome uncertainty by performing a

349 sensitivity analysis for each scenario tree. As some inputs were interdependent, we could not use the 350 AOT method and instead used the McKay method (McKay, 1995), which can be used for dependant inputs (Jacques et al., 2006), with 20 replications of Latin hypercube samples. We used the R package “sensitivity dependent" developed by Jacques, an INRA researcher.

\section{RESULTS}

3.1. Herd-level sensitivity

Table 9 summarizes the mean and 5th and 95th percentiles of the output distribution for the herd sensitivity for each protocol by herd type. The estimated mean sensitivities ranged from 20 to $60 \%$. The estimated mean herd sensitivity was higher for big herds, regardless of the protocol, production type, or herd turnover. The mean herd sensitivity was higher for low turnover than high turnover herds, regardless of the protocol and herd type. The differences of mean sensitivities between herd types for the same protocol were all significant, except for the compliant slow-path protocol between small mixed herds with a high turnover and small beef herds with a low turnover and between small mixed herds with a low turnover and small dairy herds with a low turnover. The strict protocol had the highest herd sensitivity for all herd types. The compliant slow-path protocol had the lowest mean herd sensitivity, regardless of herd type. These differences were significant with a $1 \%$ risk of error for all herd types, except for that between the two compliant protocols for small mixed herds, which were significant with a $5 \%$ risk of error. 
The mean, 5th and 95th percentiles of the output distribution of total costs by protocol and herd type are presented in Table 10. Mean costs ranged between 1,211 and 32,115 euros for the compliant slowpath protocol, 1,355 and 15,990 euros for the compliant quick-path protocol, and 2,386 and 28,394 euros for the strict protocol. Big mixed herds were found to be much more expensive to investigate than other herd types because of a larger herd size (greater than 124 animals). The strict protocol was the most expensive, regardless of herd type, except for big mixed herds. The compliant quick-path protocol was the least expensive for all big herds and small mixed herds.

\subsection{Cost-effectiveness analyses}

Figure 5 illustrates the mean cost-sensitivity index estimated for each protocol, by herd type. A high cost-sensitivity index is associated with poor cost-effectiveness of the protocol. The strict protocol was never the most cost-effective, regardless of herd type. The compliant quick path was the most costeffective protocol for big beef, big dairy, and mixed herds. The compliant slow path was the most cost-effective protocol for small beef and dairy herds. All differences were significant.

\subsubsection{Impact of inputs on herd sensitivity}

We performed sensitivity analysis of herd sensitivity output for each protocol by herd type. Mc Klay indices of the inputs greater than ten percent by herd type for each protocol are illustrated in Figure 6. This index estimates the proportion of the variation of the output caused by the variability and uncertainty of the inputs. ICCT sensitivity had the greatest influence on variations in herd sensitivity for small herds, regardless of the protocol. In big herds, ICCT sensitivity, within-herd prevalence, and herd size shared responsibility for this variation.

We performed the same sensitivity analysis for total cost output (Figure 7). For the compliant slowpath protocol, the most influential input on total costs was ICCT specificity, regardless of herd type. Size was also an influential input for small herds. For the strict protocol, the interval between the first ICCT and the second ICCT testing of the herd was the most influential for herds with a high turnover. 
variations in compensation costs. For the compliant quick-path protocol, the most influential input on

total cost variation was the interval between the first ICCT and the slaughter of ICCT non-negative animals for most of herds with a high turnover. ICCT specificity was an influential input for ten of the twelve herd types, but not for beef herd with a high turnover.

\section{DISCUSSION}

Here, we used stochastic scenario-tree modelling to estimate the herd sensitivity and cost of three mandatory investigation protocols in cattle farms for twelve herd types. We used various data sources (published studies, national databases, and expert opinions) and this method allowed us to account for the variability and the uncertainty in those data. The inputs chosen for these models were those with a documented impact on either infection or detection of bTB. Thus, we did not integrate some factors for which the impact was not sufficiently documented to allow their use. For example, the physiological status of the animals (other diseases, pregnancy, age, etc.) probably has an impact on their reactivity to the ICCT (Buddle et al., 2009; Brooks-Pollock et al., 2013; Lahuerta-Marin et al., 2016). The lack of quantitative estimations of their influence and/or the lack of consensus concerning their influence on the reactivity to ICCT led to their exclusion. Nevertheless, ICCT characteristics used in these models came from field estimations and thus the impact of these factors was partially and indirectly included in the ICCT sensitivity and specificity estimation.

The testing age limit used in the trees ( 24 months) may be surprising, as the European regulatory text recommends screening every animal over six weeks of age with the ICT. This rule is very difficult to apply in the field because young animals are too small to fit in restraining devices and thus their screening requires the help of several people. In addition, the European regulatory text allows reducing the screening frequency and increasing the age at testing in low-prevalence areas. Thus, in most regions of France, only animals older than 24 months are actually screened. 
being infected. This was not accounted for in the models because the scenario-tree method does not account for time.

Here, we only studied the cost-effectiveness of the surveillance in herds considered to either be infected or not. Further studies using the local epidemiological situation in France are necessary to assess the global cost-effectiveness of farm screening at the national or regional level.

Sensitivity analysis showed the influence of within-herd prevalence on herd sensitivity. Dairy herds are generally considered to be at greater risk of within-herd transmission because of housing conditions, but cattle are now considered to have a low risk of transmitting bTB if they are tested regularly, regardless of production type (Phillips et al., 2003) and, thus, few studies have quantitatively explored the factors that influence within-herd transmission under current conditions. In addition, the within-herd prevalence is very low in French herds, regardless of herd type, as no more than three infected animals have been found in most breakdowns (Poirier, 2017). Thus, the use of the same within-herd prevalence distribution for all herd types was probably consistent with field conditions.

ICCT sensitivity and specificity estimations showed a large distribution of values in the trees and, according to sensitivity analyses, these parameters had a significant impact on the variation of the model results (herd sensitivity and total costs). This suggests that the actual scenario trees could be improved by a more precise evaluation of these parameters, but a field evaluation of ICCT is difficult to perform and often produces biased estimations. For example, the estimation of ICCT characteristics under French field conditions (Praud and Dufour, 2016) was performed through data initially collected to evaluate IFN characteristics. ICCT-positive animals were therefore directly culled and excluded

444 from the study and it led to a selection bias probably causing an underestimation of ICCT sensitivity.

445 In addition, there is a possible recruitment bias in this study because farms with many non-negative results at the first screening or those with at least one positive ICCT did not have any particular

447 interest to participate in this program, as they could not benefit from the derogation of the sales ban 448 otherwise granted in cases of negative IFN results. These biases certainly led to an underestimation of 449 ICCT sensitivity and possibly an over-estimation of its specificity (Praud and Dufour, 2016), 
explaining why we did not use this study alone to implement our trees and combined the results of several studies to estimate the most probable value and the extremes of the ICCT characteristics. Thus, we accounted for the uncertainty concerning ICCT characteristic estimations.

We did not consider the cost of collecting blood samples in the total cost of the compliant slow-path protocol because we were unable to collect enough data to provide a realistic estimation. Therefore, the cost of the compliant slow-path protocol was underestimated, reducing the cost-effectiveness index value for this protocol. Accounting for the cost of blood sample collect could have led to an increase in the cost-effectiveness index of the compliant slow-path protocol. In this case, the compliant quickpath protocol could have become the most cost-effective protocol, regardless of herd type. In addition, in several departments of France, the stimulation of blood samples with antigens for IFN gamma testing frequently occurs in a lab different from the one reading the IFN results. This was not included in our models and would add costs (additional transport fees) to the compliant slow-path protocol, decreasing even more its cost-effectiveness.

Costs due to the slaughter of the calves of reactive cows was also not integrated into our model.

Therefore, protocol costs were probably slightly underestimated for all protocols.

Farms producing raw milk were excluded from this study. In case of suspected bTB, this type of farm cannot sell any raw milk, adding $2 €$ per day per cow (Bouveret A, 2017) to the total cost. Nevertheless, the same cost would be added per day of suspicion for a given herd type, and thus it should not change the ranking of the cost-effectiveness of the protocols.

Most of the differences in cost and sensitivity were found to be significant. This is probably due to the very high number of iterations $(10,000)$ used for the simulations.

The herd-level sensitivity values estimated in this study were between 20 and $60 \%$. Nevertheless, these estimations should not be interpreted alone to evaluate the global sensitivity of the French surveillance system. Indeed, the bTB surveillance system has other major complementary components (systematic inspection of carcasses in slaughterhouses, epidemiological investigations in farms linked to breakdowns) that improve its sensitivity. The sole purpose of this raw herd-sensitivity estimation 
was to compare the protocols for the detection of infected herds. In addition, these estimations were performed at a farm scale, with data of the average characteristics from one department. A better evaluation of the global effectiveness of bTB surveillance in France would require similar data (the proportion of each herd type, bTB prevalence, proportion of animals tested, etc.) from all departments. Further studies are ongoing to this end.

The mean herd sensitivity was higher in low-turnover than high-turnover herds, regardless of the protocol. The proportion of adults in each herd type could explain this difference. Indeed, the number of tested animals increase herd sensitivity of the test and the proportion of adults is higher in lowturnover herds (Table 1).

The costs were higher in mixed herds, regardless of the protocol, because they are much bigger than dairy and beef herds. The strict protocol was always the costliest, except for big mixed herds with a high turnover. This exception can be explained by the indirect costs (costs due to sales bans). Indeed, costs due to sales bans were always higher for the compliant slow path than for the strict protocol, but other costs in the strict protocol compensated for this difference. However, this difference in indirect costs was three times higher for big mixed herds with a high turnover (due to a higher number of animals usually sold) and thus direct costs could no longer compensate.

The strict protocol was never the most cost-effective, regardless of herd type. Its herd sensitivity may have been slightly underestimated because we did not consider that when not all animals in a herd were tested a second time with ICCT, local authorities often chose to test a second time animals that are more likely to be infected (animals of the same group of animals reactive to ICCT1). Nevertheless, according to sensitivity analyses, the proportion of animals tested by ICCT2 was not the most influential factor on herd sensitivity; in addition, the probability that these particular animals become infected still remains very low, given the very low within-herd prevalence. Improving up to $100 \%$ the percentage of animals re-tested with ICCT may seem to be a good way to improve the sensitivity of the strict protocol. With $100 \%$ of animals being re-tested, our model estimates a gain of sensitivity between $0.4 \%$ and $0.9 \%$, depending on herd type. But, it would imply to invest from 242 euros to 8,206 euros per percent gained and per herd (depending on herd type). To recommend retesting every 
animal is therefore disputable in terms of cost-effectiveness, in particular in big herds in which the additional cost will be between 1,000 and 8,000 euros per percentage of sensitivity gained and per herd.

This result suggests that compliant protocols are more cost-effective than the strict protocol approved by the European Union and thus would argue in favour of authorising compliant protocols. Nevertheless, the strict protocol is the most effective. Its lower cost-effectiveness is due to its significantly higher costs caused by a longer sales ban than the compliant quick-path protocol, and the slaughter of all animals non-negative for the first ICCT and a second ICCT on more animals than in the compliant slow-path protocol. In some context, the strict protocol could thus be preferred to compliant protocols (slow-path and quick-path) for its higher herd sensitivity, depending of the probability of infection and of the importance given to eradication purpose relatively to expenses. protocols. ICCT sensitivity was the most influential variable. This is not surprising, considering the uncertainty we had about its value.

Sensitivity analysis of the cost outcome highlights the impact of the time between protocol steps in the compliant quick-path and strict protocols. This result is logical, as there is a large difference of the time intervals between these two protocols (Table 8), but it underlines the need to reduce the authorised time intervals as much as possible to reduce indirect losses due to selling bans. Nevertheless, this reduction of time intervals is, in some cases, limited by the regulation. For example, due to the immunological effect of prior testing, regulation require a time interval between two ICCTs superior or equal to 42 days. In the compliant quick-path and compliant slow-path protocols, ICCT specificity was one of the most influential variables on variations in cost outcome, because of the substantial uncertainty we had concerning it and the impact it has on the number of slaughtered animals.

Finally, the model outcomes showed a significant difference in total costs and herd sensitivity between the three protocols studied. These three protocols are therefore not equivalent and seeking the most cost-effective, depending on the context, is logical. The cost-effectiveness index calculated for each 
protocol by herd type in this study provides data to help authorities choose the best protocol, as it highlights the most cost-effective protocol for each herd type. Nevertheless, cost-effectiveness is not the only indicator that should be considered. Indeed, our estimation did not include sociological factors, such as the acceptability of measures that can affect the effectiveness of a surveillance system (Broughan et al., 2016; Calba et al., 2016). For example, some veterinarians may not notify to

536 authorities some non-negative results to ICCT, to avoid their customer's farm to be considered as bTB 537 suspect. Acceptability issues mainly come from the sales ban for a suspect herd (or blocking period).

538 Indeed, such sales bans can be difficult for the farmer to tolerate and cause him economic loss. The non-notification by the veterinarian to the authorities of non-negative ICCTs could be due to various causes which will be useful to investigate. These can include the frequency of falsely positive ICT results, as notification has heavy economic repercussions (Calba et al., 2016), or the close relationship between veterinarians and farmers, as the veterinarian responsible for this mandatory measure is the

543 habitual veterinarian of the farm. In addition, ICCT characteristics depend on how the test is

544 administered (Tefka, 1986; Monaghan et al., 1994; Schiller et al., 2010), which may vary from one

545 veterinarian to another (Humblet et al., 2011). The inclusion of these factors in the scenario trees

546 could help to achieve a more accurate cost-effectiveness estimation of these protocols, depending on

547 the sociological context considered.

\section{CONCLUSION}

550 We presented the use of stochastic scenario-tree modelling to estimate the herd sensitivity and cost of three protocols used to investigate suspected bTB in French cattle farms by herd type (herd production, size, and turnover). This study identified the most cost-effective protocol for each defined type of herd. It suggests that, regardless of herd type and based on herd sensitivity and cost, the strict protocol is never the most cost-effective, due to higher costs than for the other protocols, despite better

555 herd sensitivity. This comparison of protocols could help authorities to choose the best protocol,

556 depending on the herd characteristics. Nevertheless, there is a need to account for sociological factors, 
such as the acceptability of the measures for veterinarians, which may change the effectiveness estimate of these protocols and their cost-effectiveness ranking.

\section{ACKNOWLEDGEMENTS}

The authors want to thank the French Ministry of Agriculture and the French Agency for Food, Environmental, and Occupational Health and Safety for providing access to the SIGAL and BDNI data. We also thank the ADILVA for their help in administrating the survey among departmental analysis laboratories; M-L Boschiroli, director of the national reference laboratory for bovine tuberculosis, for providing his insight concerning laboratory tests; and the regional and national bTB coordinators for their help, especially F. Chevalier for his validation of some of the parameters. Finally, we thank William Hempel from Alex Edelman and Associates for the English proofreading of the manuscript.

\section{FUNDING}

The French Ministry of Agriculture and the French Agency for Food, Environmental, and Occupational Health and Safety supported this work.

\section{BIBLIOGRAPHY}

Brooks-Pollock, E., Conlan, A.J., Mitchell, A.P., Blackwell, R., McKinley, T.J., Wood, J.L., 2013. Age-dependent patterns of bovine tuberculosis in cattle. Vet. Res. 44, 97.

Broughan, J.M., Maye, D., Carmody, P., Brunton, L.A., Ashton, A., Wint, W., Alexander, N., Naylor, R., Ward, K., Goodchild, A.V., Hinchliffe, S., Eglin, R.D., Upton, P., Nicholson, R., Enticott, G., 2016. Farm characteristics and farmer perceptions associated with bovine tuberculosis incidents in areas of emerging endemic spread. Prev. Vet. Med. 129, 88-98.

Bouveret A, 2017. Evaluation coût efficacité préliminaire de la composante de surveillance de la tuberculose bovine en élevage en France. Veterinary thesis, ENVA. 145p. 
Buczinski B., Reuillon J.-L., 2015. Chiffrage des pertes économiques dans une exploitation d'élevage bovin lait ou bovin viande suite au blocage de ses ventes. Report $n^{\circ} 0015502034$ of the French livestock institute. 14p.

Buddle, B., Livingstone, P., de Lisle, G., 2009. Advances in ante-mortem diagnosis of tuberculosis in cattle. N. Z. Vet. J. 57, 173-180.

Calba, C., Goutard, F.L., Vanholme, L., Antoine-Moussiaux, N., Hendrikx, P., Saegerman, C., 2016. The Added-Value of Using Participatory Approaches to Assess the Acceptability of Surveillance Systems: The Case of Bovine Tuberculosis in Belgium. PLoS ONE 11.

Courcoul, A., BOSCHIROLI, M.-L., 2013. Rapport d'appui scientifique et technique : Demande d'Appui Scientifique sur le protocole de diagnostic de la tuberculose bovine par PCR. Report of the French Agency for Food, Environmental and Occupational Health and Safety. 40p.

Courcoul, A., Moyen, J.-L., Brugère, L., Faye, S., Hénault, S., Gares, H., Boschiroli, M.-L., 2014. Estimation of Sensitivity and Specificity of Bacteriology, Histopathology and PCR for the Confirmatory Diagnosis of Bovine Tuberculosis Using Latent Class Analysis. PLoS ONE. 9.

EFSA, 2012. Technical meeting of the EFSA Scientific Network for Risk assessment in Animal Health and Welfare - Bovine Tuberculosis Testing. EFSA Support. Publ. 9.

Gardner, I.A., Stryhn, H., Lind, P., Collins, M.T., 2000. Conditional dependence between tests affects the diagnosis and surveillance of animal diseases. Prev. Vet. Med. 45, 107-122.

Hadorn, D.C., Racloz, V., Schwermer, H., Stärk, K.D.C., 2009. Establishing a cost-effective national surveillance system for Bluetongue using scenario tree modelling. Vet. Res. 57.

Humblet, M.-F., Walravens, K., Salandre, O., Boschiroli, M.L., Gilbert, M., Berkvens, D., FauvilleDufaux, M., Godfroid, J., Dufey, J., Raskin, A., Vanholme, L., Saegerman, C., 2011. Monitoring of the intra-dermal tuberculosis skin test performed by Belgian field practitioners. Res. Vet. Sci. 91, 199-207.

Jacques, J., Lavergne, C., Devictor, N., 2006. Sensitivity analysis in presence of model uncertainty and correlated inputs. Reliab. Eng. Syst. Saf. 91, 1126-1134. 
Lahuerta-Marin, A., McNair, J., Skuce, R., McBride, S., Allen, M., Strain, S.A.J., Menzies, F.D., McDowell, S.J.W., Byrne, A.W., 2016. Risk factors for failure to detect bovine tuberculosis in cattle from infected herds across Northern Ireland (2004-2010). Res. Vet. Sci. 107, 233-239.

Mackay, I.M., 2004. Real-time PCR in the microbiology laboratory. Clin. Microbiol. Infect. 10, 190212.

Martin, P.A.J., Cameron, A.R., Barfod, K., Sergeant, E.S.G., Greiner, M., 2007. Demonstrating freedom from disease using multiple complex data sources 2: Case study-Classical swine fever in Denmark. Prev. Vet. Med. 79, 98-115.

McKay, M.D., 1995. Evaluating prediction uncertainty. Technical report nº. NUREG/CR--6311, LA-12915-MS, 29432. Available at: https://doi.org/10.2172/29432.

Monaghan, M.L., Doherty, M.L., Collins, J.D., Kazda, J.F., Quinn, P.J., 1994. The tuberculin test. Vet. Microbiol. 40, 111-124.

Müller, B., Dürr, S., Alonso, S., Hattendorf, J., Laisse, C.J.M., Parsons, S.D.C., van Helden, P.D., Zinsstag, J., 2013. Zoonotic mycobacterium bovis-induced Tuberculosis in Humans. Emerg. Infect. Dis. 19, 899-908.

Perrin, J.-B., Ducrot, C., Vinard, J.-L., Morignat, E., Calavas, D., Hendrikx, P., 2012. Assessment of the utility of routinely collected cattle census and disposal data for syndromic surveillance. Prev. Vet. Med. 105, 244-252.

Phillips, C.J., Foster, C.R., Morris, P., Teverson, R., 2003. The transmission of Mycobacterium bovis infection to cattle. Res. Vet. Sci. 74, 1-15.

Poirier, V., 2017. Elevages foyers de tuberculose bovine concernés par l'abattage partiel en France depuis son autorisation sur tout le territoire en 2014 : typologie des élevages concernés, efficacité du dispositif. Veterinary thesis, ENVA. 98p.

Praud, A., Dufour, B., 2016. Rapport sur l'analyse des données collectées dans le cadre du protocole expérimental d'évaluation de l'IFN pour le dépistage de la tuberculose bovine et dans les élevages en suivi renforcé de 2013 à 2015. Report, ENVA.

Schiller, I., Oesch, B., Vordermeier, H.M., Palmer, M.V., Harris, B.N., Orloski, K.A., Buddle, B.M., Thacker, T.C., Lyashchenko, K.P., Waters, W.R., 2010. Bovine Tuberculosis: A Review of 

and Eradication. Transbound. Emerg. Dis. 57, 205-220.

639 Sicard, O., 2013. La loi normale asymétrique, définition, premières propriétés, estimation des paramètres. French research institute of mathematical education, informatics laboratory of Réunion. Avaible at: http://irem.univreunion.fr/IMG/pdf/normale_asymetrique_sicard_2013.pdf

Tefka, N., 1986. Contribution à l'étude de la valeur des techniques d'intradermotuberculinations dans le diagnostique de la tuberculose bovine selon le contexte épidémiologique. Veterinary thesis, ENVA.

646 Thoen, C., LoBue, P., de Kantor, I., 2006. The importance of Mycobacterium bovis as a zoonosis. 


\section{$649 \quad$ Figure captions}

650 Figure 1. Scenario tree illustrating the French compliant quick-path protocol of bTB surveillance

651 Figure 2. Scenario tree illustrating the French strict protocol of bTB surveillance

652 Figure 3. Scenario tree illustrating the French compliant slow-path protocol of bTB surveillance

653 Figure 4. Scenario tree illustrating the diagnosis of bTB in a slaughtered animal

654 Figure 5. Cost-sensitivity index for the three studied protocols by herd type (10,000 iterations)

655 Figure 6. Sensitivity analysis index above 10\% (Mc Kay method) for the output "herd sensitivity" by

656 herd type for each protocol

657 Figure 7: Sensitivity analysis index of greater than 10\% (Mc Kay method) for the output "total costs"

658 by herd type for each protocol 


\section{Tables}

Table 1. Estimated distributions of the proportion of adult and young cattle by herd type (production type, size, and herd turnover)

\begin{tabular}{|c|c|c|c|c|}
\hline \multirow{2}{*}{ Production type } & \multirow{2}{*}{ Herd size category } & \multirow{2}{*}{ Herd turnover } & \multicolumn{2}{|c|}{ Age } \\
\hline & & & $\geq 24$ months & $<24$ months \\
\hline \multirow{4}{*}{ Dairy farm } & \multirow{2}{*}{ small } & $<40 \%$ & $\mathrm{~N}(0.659,0.00207)$ & Complementary to one \\
\hline & & $\geq 40 \%$ & $\mathrm{~N}(0.473,0.0127)$ & Complementary to one \\
\hline & \multirow{2}{*}{ big } & $<40 \%$ & $\mathrm{~N}(0.625,0.00248)$ & Complementary to one \\
\hline & & $\geq 40 \%$ & $\mathrm{~N}(0.581,0.0296)$ & Complementary to one \\
\hline \multirow{4}{*}{ Beef farm } & \multirow{2}{*}{ small } & $<40 \%$ & $\mathrm{~N}(0.578,0.00180)$ & Complementary to one \\
\hline & & $\geq 40 \%$ & $\mathrm{~N}(0.491,0.00683)$ & Complementary to one \\
\hline & \multirow{2}{*}{ big } & $<40 \%$ & $\mathrm{~N}(0.546,0.00122)$ & Complementary to one \\
\hline & & $\geq 40 \%$ & $\mathrm{~N}(0.212,0.00719$ & Complementary to one \\
\hline \multirow{4}{*}{ Mixed farm } & \multirow{2}{*}{ small } & $<40 \%$ & $\mathrm{~N}(0.617,0.00212)$ & Complementary to one \\
\hline & & $\geq 40 \%$ & $\mathrm{~N}(0.553,0.0116)$ & Complementary to one \\
\hline & \multirow{2}{*}{ big } & $<40 \%$ & $\mathrm{~N}(0.581,0.00246)$ & Complementary to one \\
\hline & & $\geq 40 \%$ & $\mathrm{~N}(0.537,0.0144)$ & Complementary to one \\
\hline
\end{tabular}

Legend: Normal(mean, SD)

Table 2. Values of the parameters of the distribution laws used to model ICCT sensitivity and specificity and estimated through published studies

\begin{tabular}{|c|c|c|c|c|}
\hline Test & Characteristic & $\begin{array}{r}\text { Normal asym } \\
\text { parame }\end{array}$ & tric law & Sources \\
\hline \multirow{6}{*}{$\begin{array}{l}\text { First ICCT } \\
\text { (ICCT1) }\end{array}$} & \multirow{3}{*}{ sensitivity } & CI lower limit & $43 \%$ & Praud and Dufour, 2016 \\
\hline & & Mean & $74 \%$ & $\begin{array}{l}\text { Mean of estimations from de la Rua-Domenech et al. } \\
\text { 2006a, Clegg et al. 2011, Praud and Dufour, 2016, } \\
\text { Pucken et al. 2017, Nuñez-Garcia et al. } 2017\end{array}$ \\
\hline & & CI upper limit & $95 \%$ & Pucken et al., 2017 \\
\hline & \multirow{3}{*}{ specificity } & CI lower limit & $80 \%$ & Praud and Dufour, 2016 and Clegg et al., 2011 \\
\hline & & Mean & $99 \%$ & $\begin{array}{c}\text { Mean of estimations from Praud and Dufour, } 2016 \\
\text { and Clegg et al., } 2011\end{array}$ \\
\hline & & CI upper limit & $100 \%$ & Praud and Dufour, 2016 and Clegg et al., 2011 \\
\hline \multirow{6}{*}{$\begin{array}{c}\text { ICCT } \\
\text { conditional to } \\
\text { a first non- } \\
\text { negative } \\
\text { result to } \\
\text { ICCT } \\
\text { (ICCT2) }\end{array}$} & \multirow{3}{*}{ sensitivity } & CI lower limit & $43 \%$ & Praud and Dufour, 2016 \\
\hline & & Mean & $73 \%$ & $\begin{array}{l}\text { Mean of estimations from de la Rua-Domenech et } \\
\text { al., 2006; Praud Anne et al., 2015; Pucken et al., } \\
2017\end{array}$ \\
\hline & & CI upper limit & $95 \%$ & Pucken et al., 2017 \\
\hline & \multirow{3}{*}{ specificity } & CI lower limit & $87 \%$ & \multirow{3}{*}{ Praud and Dufour, 2016} \\
\hline & & Mean & $89 \%$ & \\
\hline & & CI upper limit & $90 \%$ & \\
\hline
\end{tabular}

CI: $95 \%$ confidence interval 
Table 3. Estimated distributions of the probability of occurrence of each possible IFN results by animal status for bTB (Praud and Dufour, 2016)

\begin{tabular}{ccc}
\hline \multirow{2}{*}{ IFN result } & \multicolumn{2}{c}{ bTB animal status } \\
\cline { 2 - 3 } & Infected & Uninfected \\
\hline Negative & $\mathrm{N}(0.035,0.020)$ & $\mathrm{Neg}=\mathrm{N}(0.458,0.00969)^{*}$ \\
\hline Inconclusive & Complementary to one & $\mathrm{N}(0.818,0.00765)^{* * *}$ Neg \\
\hline Positive & $\mathrm{N}(0.93,0.0255)^{* *}$ & Complementary to one \\
\hline
\end{tabular}

Legend: Normal(mean, SD)

*Specificity according to EU standards (the only animals considered to be negative are those with a negative result (exclusion of inconclusive results))

** Raw sensitivity (only the animals with a positive result are considered to be positive (exclusion of inconclusive results))

*** Raw specificity (animals with negative or inconclusive results are considered to be negative) 


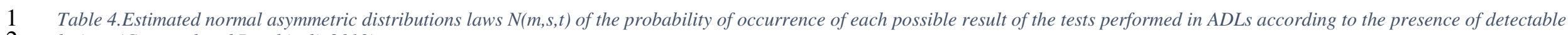
lesions (Courcoul and Boschiroli, 2013)

\begin{tabular}{|c|c|c|c|c|c|}
\hline \multirow{2}{*}{ Analysis } & \multirow{2}{*}{ Result } & \multicolumn{2}{|l|}{ Infected animal } & \multicolumn{2}{|c|}{ Uninfected animal } \\
\hline & & With lesions & Without lesions & With lesions & Without lesions \\
\hline \multirow{3}{*}{ PCR } & Positive & \multirow{2}{*}{$\mathrm{x}=\mathrm{N}(0.887,0.825,0.923)$} & $\mathrm{p}_{1=} \mathrm{N}(0.909,0.873,0.943)$ & 0 & $1-\mathrm{p}_{3}$ \\
\hline & Doubtful $^{(1)}$ & & 0 & $1-\mathrm{p}_{2}$ & 0 \\
\hline & Negative & $1-\mathrm{x}$ & $1-\mathrm{p}_{1}$ & $\begin{array}{c}\mathrm{p}_{2}=\mathrm{N}(0.970,0.943 \\
0.990)\end{array}$ & $\mathrm{p}_{3}=\mathrm{N}(0.998,0.994,1)$ \\
\hline \multirow{2}{*}{ Bacteriology } & Positive & $\mathrm{p}_{4}=\mathrm{N}(0.781,0.729,0.828)$ & $\mathrm{p}_{5}=\mathrm{N}(0.792,0.744,0.843)$ & $1-\mathrm{p}_{6}$ & $1-\mathrm{p}_{7}$ \\
\hline & Negative & $1-\mathrm{p}_{4}$ & $1-\mathrm{p}_{5}$ & $\mathrm{p}_{6}=\mathrm{N}(0.991,0.971,1)$ & $\mathrm{p}_{7}=\mathrm{N}(0.999,0.997,1)$ \\
\hline \multirow[b]{2}{*}{ Histology } & Positive & $\mathrm{p}_{8}=\mathrm{N}(0.936,0.899,0.969)$ & I & $1-\mathrm{p}_{9}$ & / \\
\hline & Negative & $1-\mathrm{p}_{8}$ & / & $\begin{array}{c}\mathrm{p}_{9}=\mathrm{N}(0.833,0.787 \\
0.876)\end{array}$ & / \\
\hline
\end{tabular}

3 Legend: $\mathrm{N}(\mathrm{m}, \mathrm{s}, \mathrm{t})=$ normal asymmetric distribution law $(\mathrm{m}=$ mean, $\mathrm{s}=$ lower value of CI95\%, $\mathrm{t}=\mathrm{upper}$ value of the CI95\%); U(min, max $)=\mathrm{uniform}$

4 distribution law.

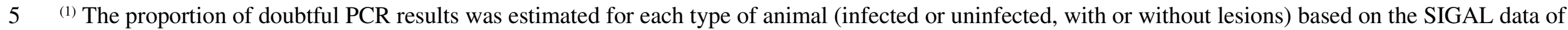

6 2016. In these data, there were no doubtful PCR results for any animals without lesions (infected (74 animals) or uninfected (3,438 animals)). Of the 888

7 uninfected animals with lesion(s), only one animal showed a doubtful result (equal to $100 \%$ of the positive or doubtful results). In $2016,50 \%$ of PCR non-

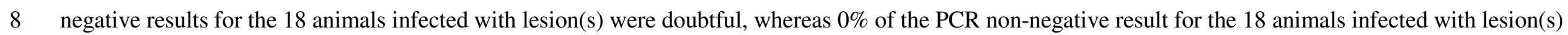

9 in 2015 were doubtful. 
Table 5. Estimated distributions of herd size (number of animals) by herd type (production type, size, and herd turnover)

\begin{tabular}{|c|c|c|c|c|}
\hline Production type & Herd size category & $\begin{array}{c}\text { Herd } \\
\text { turnover }\end{array}$ & Herd size & Comments \\
\hline \multirow{4}{*}{ Dairy farm } & \multirow{2}{*}{ small } & $<40 \%$ & $\mathrm{~N}(44.24,15.04)^{(1)}$ & \multirow{2}{*}{ Restrained between 1 and $74^{(2)}$} \\
\hline & & $\geq 40 \%$ & $\mathrm{~N}(28.88,16.25)^{(1)}$ & \\
\hline & \multirow{2}{*}{ big } & $<40 \%$ & $\mathrm{~N}(98.86,26.13)^{(1)}$ & \multirow{2}{*}{ Restrained to be higher than $74^{(3)}$} \\
\hline & & $\geq 40 \%$ & $\mathrm{~N}(80.50,5.50)^{(1)}$ & \\
\hline \multirow{4}{*}{ Beef farm } & \multirow{2}{*}{ small } & $<40 \%$ & $\mathrm{~N}(39.27,15.55)^{(1)}$ & \multirow{2}{*}{ Restrained between 1 and $66^{(2)}$} \\
\hline & & $\geq 40 \%$ & $\mathrm{~N}(34.38,17.92)^{(1)}$ & \\
\hline & \multirow{2}{*}{ big } & $<40 \%$ & $\mathrm{~N}(106.28,38.26)^{(1)}$ & \multirow{2}{*}{ Restrained to be higher than $66^{(3)}$} \\
\hline & & $\geq 40 \%$ & $\mathrm{~N}(106.70,43.70)^{(1)}$ & \\
\hline \multirow{4}{*}{ Mixed farm } & \multirow{2}{*}{ small } & $<40 \%$ & $\mathrm{~N}(79.01,22.60)^{(1)}$ & \multirow{2}{*}{ Restrained between 1 and $124^{(2)}$} \\
\hline & & $\geq 40 \%$ & $\mathrm{~N}(66.67,23.51)^{(1)}$ & \\
\hline & \multirow{2}{*}{ big } & $<40 \%$ & $\mathrm{~N}(164.32,38.60)^{(1)}$ & \multirow{2}{*}{ Restrained to be higher than $124^{(3)}$} \\
\hline & & $\geq 40 \%$ & $\mathrm{~N}(156.75,38.71)^{(1)}$ & \\
\hline
\end{tabular}

\section{Legend:}

$13 \operatorname{Normal}($ mean, SD)

14 (1) The number is rounded up to the next highest integer; parameters were defined to fit the BDIN data.

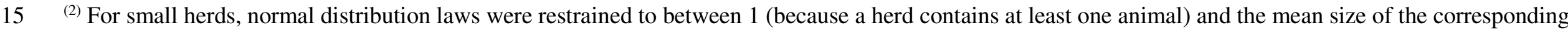

16 type of herd (dairy: 74, beef: 66, or mixed: 124 animals) which was used as a limit to define the size categories of the herds.

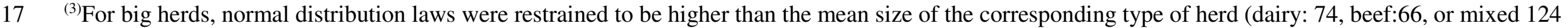
animals) which was used as a limit to define the size categories of herds. 
21 Table 6. Price data of the laboratory test performed in bTB surveillance from the ADL survey and distributions used in our scenario trees to model the cost of each test

\begin{tabular}{|c|c|c|c|c|c|c|c|c|}
\hline \multirow[t]{2}{*}{ Analysis } & \multicolumn{4}{|c|}{$\begin{array}{c}\text { Price data indicated by ADL } \\
\text { surveyed } \\
\text { (in } € / \text { sample })\end{array}$} & \multirow[t]{2}{*}{$\mathbf{N}$} & \multirow[t]{2}{*}{ Covered by the price } & \multirow[t]{2}{*}{$\begin{array}{l}\text { Not covered } \\
\text { by the price }\end{array}$} & \multirow[t]{2}{*}{ Description in the study models } \\
\hline & Min & Median & Mean & Max & & & & \\
\hline IFN $\gamma$ & 34.00 & 53.15 & 51.76 & 67.63 & 6 & IFN Analysis + administrative fees & Sample collect & $\operatorname{PERT}(\min =34, \operatorname{mode}=53, \max =68)$ \\
\hline PCR & 50.00 & 74.28 & 75.17 & 114.00 & 10 & PCR analyses + administrative fees & & $\operatorname{PERT}(\min =50, \operatorname{mode}=74, \max =114)$ \\
\hline $\begin{array}{l}\text { Bacteriology (culture of a } \\
\text { mix of the three samples if } \\
\text { there are no lesions) }\end{array}$ & 35.36 & 68.81 & 75.14 & 185.00 & 10 & $\begin{array}{l}\text { Mix processing }+ \text { plating }+ \text { bacterial } \\
\text { culture }\end{array}$ & & $\operatorname{PERT}(\min =35$, mode $=69, \max =185)$ \\
\hline $\begin{array}{l}\text { Individual bacteriology (for } \\
\text { each sample if there are } \\
\text { lesions) }\end{array}$ & 35.36 & 58.13 & 62.04 & 90.00 & 10 & Plating + bacterial culture & & $\operatorname{PERT}(\min =35, \operatorname{mode}=58, \max =90)$ \\
\hline $\begin{array}{l}\text { Transport fees of PCR }+ \\
\text { DNA to the NRL }\end{array}$ & 19.23 & 22.19 & 27.88 & 45.00 & 5 & & & $\operatorname{PERT}(\min =19, \operatorname{mode}=28, \max =45)$ \\
\hline $\begin{array}{c}\text { Transport fees of bacterial } \\
\text { strain or doubtful PCR } \\
\text { sample }\end{array}$ & 80 & & 460 & 870 & 7 & & & $\begin{array}{c}\operatorname{PERT}(\min =80, \text { mode }=460, \\
\max =870)^{(1)}\end{array}$ \\
\hline $\begin{array}{l}\text { Sample processing for } \\
\text { histology }\end{array}$ & 0 & 10.92 & 10.73 & 20.39 & 8 & Sample processing & & PERT $(\min =0, \operatorname{mode}=11, \max =20)$ \\
\hline $\begin{array}{l}\text { Transport fees for a sample } \\
\text { to the ADL for histology }\end{array}$ & 10.00 & 15.53 & 18.9 & 31.70 & 6 & & & $\operatorname{PERT}(\min =10, \operatorname{mode}=16, \max =32)$ \\
\hline Histology & 65.46 & 65.73 & 65.73 & 66.00 & 2 & Histological analysis & $\begin{array}{c}\text { Sample } \\
\text { processing }\end{array}$ & 66 \\
\hline
\end{tabular}

Legend: $\mathrm{N}=$ number of prices considered, $\mathrm{Min}=$ minimum price reported, $\mathrm{Max}=$ maximum price reported

(1) Multiplied by 0.25 if it is a bacterial strain, because several bacterial strains (a mean of four, personal communication Boschiroli M.L. NRL) are usually 


\section{Production type}

\begin{tabular}{|c|c|c|c|}
\hline Animal & Dairy & Beef & Mixed \\
\hline Female $<24$ months & $\operatorname{PERT}(\min =2.87, \operatorname{mode}=2.87, \max =3.79)$ & $\mathrm{U}(1, \min =2.04, \max =2.09)$ & $\operatorname{PERT}(\min =1.88, \operatorname{mode}=2.47, \max =3.79)$ \\
\hline Female $\geq 24$ months & $\operatorname{PERT}(\min =3.18, \operatorname{mode}=3.18, \max =4.18)$ & $\mathrm{U}(1, \min =3.29, \max =3.7)$ & $\operatorname{PERT}(\min =3.18, \operatorname{mode}=3.38, \max =4.18)$ \\
\hline Male dairy calves $^{(1)}$ & $\operatorname{PERT}(\min =103.66, \operatorname{mode}=103.66, \max =122.06)^{(1)}$ & l & $\operatorname{PERT}(\min =103.66, \operatorname{mode}=103.66, \max =122.06)^{(1)}$ \\
\hline Male $\geq 6$ months & 1 & $\mathrm{U}(1, \min =3.14, \max =3.32)$ & $\mathrm{U}(1, \min =3.14, \max =3.32)$ \\
\hline Male $<6$ months & l & $\mathrm{U}(1, \min =1.30, \max =1.42)$ & $\mathrm{U}(1, \min =1.30, \max =1.42)$ \\
\hline
\end{tabular}

PERT = PERT distribution law; U=uniform distribution law

${ }^{(1)}$ The cost for male dairy calves is in $€$ per animal for the entire sales interdiction period. It corresponds to the impossibility of selling male calves at the required age; the farmers must then sell the calves as fat calves fed whole milk.

Table 8. Distributions used to model the interval (in days) between each protocol step for each studied protocol (source: SIGAL)

\begin{tabular}{|c|c|c|}
\hline Protocol & Interval (one step - another step) & Distribution law used \\
\hline \multirow{3}{*}{ Strict } & First ICCT result - slaughter of non-negative animals by the first ICCT & $\operatorname{PERT}(\min =1, \operatorname{mode}=21, \max =150)$ \\
\hline & First ICCT results - second ICCT & $\operatorname{PERT}(\min =40, \operatorname{mode}=48, \max =133)$ \\
\hline & Second ICCT- slaughter of non-negative animals by the second ICCT & $\operatorname{PERT}(\min =3, \operatorname{mode}=22, \max =69)$ \\
\hline Compliant quick path & First ICCT result - slaughter of non-negative animals by the first ICCT & $\operatorname{PERT}(\min =4, \operatorname{mode}=35, \max =65)$ \\
\hline \multirow{4}{*}{ compliant slow path } & First ICCT result - slaughter of animals with positive IFN results & \multirow[b]{2}{*}{$\operatorname{PERT}(\min =3, \operatorname{mode}=24, \max =136)$} \\
\hline & $\begin{array}{l}\text { Slaughter of animals with positive IFN results - slaughter of animals with non-negative results by the } \\
\text { first ICCT and non-positive IFN results }\end{array}$ & \\
\hline & First ICCT results - second ICCT & $\operatorname{PERT}(\min =33, \operatorname{mode}=41, \max =130)$ \\
\hline & Second ICCT - slaughter of non-negative animals by the second ICCT & PERT $(\min =8, \operatorname{mode}=22, \max =154)$ \\
\hline
\end{tabular}




\begin{tabular}{|c|c|c|c|c|c|c|c|c|}
\hline \multirow[t]{2}{*}{ Production type } & \multirow[t]{2}{*}{ Herd size category } & \multirow[t]{2}{*}{ Herd turnover } & \multicolumn{2}{|c|}{ Compliant slow path protocol } & \multicolumn{2}{|c|}{ Compliant quick path protocol } & \multicolumn{2}{|c|}{ Strict protocol } \\
\hline & & & Mean $(\%)$ & CI95\% (\%) & Mean $(\%)$ & CI95\% (\%) & Mean $(\%)$ & $\mathrm{CI} 95 \%(\%)$ \\
\hline \multirow[t]{4}{*}{ Dairy farm } & \multirow{2}{*}{$\begin{array}{c}\text { small } \\
(<74 \text { adults })\end{array}$} & $<40 \%$ & 42.7 & $42.5-42.8$ & 44.2 & 44.1-44.4 & 47.4 & $47.2-47.5$ \\
\hline & & $\geq 40 \%$ & 30.8 & $30.7-30.9$ & 31.6 & $31.5-31.7$ & 34.3 & $34.2-34.4$ \\
\hline & \multirow{2}{*}{$\begin{array}{c}\text { big } \\
(\geq 74 \text { adults })\end{array}$} & $<40 \%$ & 49.0 & $48.7-49.2$ & 51.6 & $51.3-51.8$ & 52.3 & $52.1-52.6$ \\
\hline & & $\geq 40 \%$ & 40.4 & $40.3-40.6$ & 42.4 & $42.3-42.6$ & 43.7 & $43.5-43.9$ \\
\hline \multirow[t]{4}{*}{ Beef farm } & \multirow{2}{*}{$\begin{array}{c}\text { small } \\
(<66 \text { adults })\end{array}$} & $<40 \%$ & 37.5 & $37.4-37.7$ & 38.7 & $38.6-38.9$ & 41.8 & $41.7-41.9$ \\
\hline & & $\geq 40 \%$ & 32.0 & $31.9-32.1$ & 32.9 & $32.7-33.0$ & 35.6 & $35.5-35.7$ \\
\hline & \multirow{2}{*}{$\begin{array}{c}\text { big } \\
(\geq 66 \text { adults })\end{array}$} & $<40 \%$ & 46.0 & $45.7-46.2$ & 48.2 & $48.0-48.5$ & 49.1 & $48.8-49.3$ \\
\hline & & $\geq 40 \%$ & 20.2 & $20.0-20.3$ & 20.8 & $20.7-21.0$ & 21.7 & $21.5-21.8$ \\
\hline \multirow[t]{4}{*}{ Mixed farm } & \multirow{2}{*}{$\begin{array}{c}\text { small } \\
(<124 \text { adults })\end{array}$} & $<40 \%$ & 43.0 & $42.8-43.2$ & 45.1 & $44.9-45.3$ & 46.6 & $46.5-46.8$ \\
\hline & & $\geq 40 \%$ & 37.7 & $37.5-37.8$ & 39.3 & $39.1-39.5$ & 41.2 & $41.1-41.4$ \\
\hline & \multirow{2}{*}{$\begin{array}{c}\text { big } \\
(\geq 124 \text { adults })\end{array}$} & $<40 \%$ & 61.0 & $60.7-61.3$ & 63.9 & $63.7-64.2$ & 64.2 & 63.9-64.4 \\
\hline & & $\geq 40 \%$ & 56.8 & $56.6-57.1$ & 59.6 & 59.4-59.9 & 59.9 & $59.7-60.2$ \\
\hline
\end{tabular}




\begin{tabular}{|c|c|c|c|c|c|c|c|c|}
\hline \multirow[t]{2}{*}{ Production type } & \multirow[t]{2}{*}{ Herd size category } & \multirow[t]{2}{*}{ Herd turnover } & \multicolumn{2}{|c|}{ Compliant slow path protocol } & \multicolumn{2}{|c|}{ Compliant quick path protocol } & \multicolumn{2}{|c|}{ Strict protocol } \\
\hline & & & Mean & CI95\% & Mean & CI95\% & Mean & CI95\% \\
\hline \multirow[t]{4}{*}{ Dairy farm } & \multirow{2}{*}{$\begin{array}{c}\text { small } \\
(<74 \text { adults })\end{array}$} & $<40 \%$ & 2,393 & $2,354-2,432$ & 2,702 & $2,670-2,735$ & 4,480 & $4,426-4,533$ \\
\hline & & $\geq 40 \%$ & 2,459 & $2,422-2,497$ & 3,208 & $3,183-3,234$ & 5,591 & $5,543-5,639$ \\
\hline & \multirow{2}{*}{$\begin{array}{c}\text { big } \\
(\geq 74 \text { adults })\end{array}$} & $<40 \%$ & 6,988 & $6,884-7,092$ & 5,905 & $5,836-5,975$ & 8,949 & $8,851-9,047$ \\
\hline & & $\geq 40 \%$ & 9,263 & $9,120-9,405$ & 6,948 & $6,892-7,005$ & 11,983 & $11,889-12,077$ \\
\hline \multirow[t]{4}{*}{ Beef farm } & \multirow{2}{*}{$\begin{array}{c}\text { small } \\
(<66 \text { adults })\end{array}$} & $<40 \%$ & 1,211 & $1,190-1,232$ & 1,355 & $1,340-1,370$ & 2,386 & $2,359-2,412$ \\
\hline & & $\geq 40 \%$ & 2,351 & $2,307-2,395$ & 2,933 & $2,907-2,959$ & 5,875 & $5,822-5,928$ \\
\hline & \multirow{2}{*}{$\begin{array}{c}\text { big } \\
(\geq 66 \text { adults })\end{array}$} & $<40 \%$ & 5,370 & $5,273-5,467$ & 3,569 & $3,528-3,609$ & 6,021 & $5,960-6,081$ \\
\hline & & $\geq 40 \%$ & 7,470 & $7,314-7,625$ & 6,948 & $6,886-7,011$ & 14,051 & $13,922-14,180$ \\
\hline \multirow[t]{3}{*}{ Mixed farm } & $\begin{array}{c}\text { small } \\
(<124 \text { adults })\end{array}$ & $<40 \%$ & 4,950 & $4,866-5,033$ & 4,303 & $4,249-4,356$ & 7,048 & $6,967-7,129$ \\
\hline & \multirow{2}{*}{$\begin{array}{c}\text { big } \\
(\geq 124 \text { adults })\end{array}$} & $<40 \%$ & 13,065 & $12,864-13,266$ & 8,692 & $8,584-8,800$ & 13,179 & $13,033-13,324$ \\
\hline & & $\geq 40 \%$ & 32,688 & $32,115-33,261$ & 15,990 & $15,857-16,122$ & 28,619 & $28,394-28,844$ \\
\hline
\end{tabular}




\section{Appendix}

Appendix 1. Description of the normal asymmetric distribution law (Sicard, 2013)

If $X$ follows an asymmetric normal law $\mathrm{N}(\mathrm{m}, \mathrm{s}, \mathrm{t})$, then the interval containing $95 \%$ of $\mathrm{X}$ values is [m-

$1.96 * \mathrm{~s} ; \mathrm{m}+\& .96 * \mathrm{t}]$.

In addition, if $\mathrm{U}$ follows a Bernoulli distribution law of parameter $\mathrm{s} /(\mathrm{s}+\mathrm{t}), \mathrm{Xs}$ a normal distribution law $\mathrm{N}(0, \mathrm{~s})$ and $\mathrm{Xt}$ a normal distribution law $\mathrm{N}(0, \mathrm{t})$, then $\mathrm{X}^{\prime}=\mathrm{U}^{*}|\mathrm{Xs}|+(1-\mathrm{U})^{*}|\mathrm{Xt}|$ follows a normal symmetric law $\mathrm{N}(0, \mathrm{~s}, \mathrm{t})$ and $\mathrm{X}=\mathrm{X}^{\prime}+\mathrm{m}$ follows a normal asymmetric law $\mathrm{N}(\mathrm{m}, \mathrm{s}, \mathrm{t})$.

An $\mathrm{R}$ function based on this definition was created to simulate a normal asymmetric distribution law as described below:

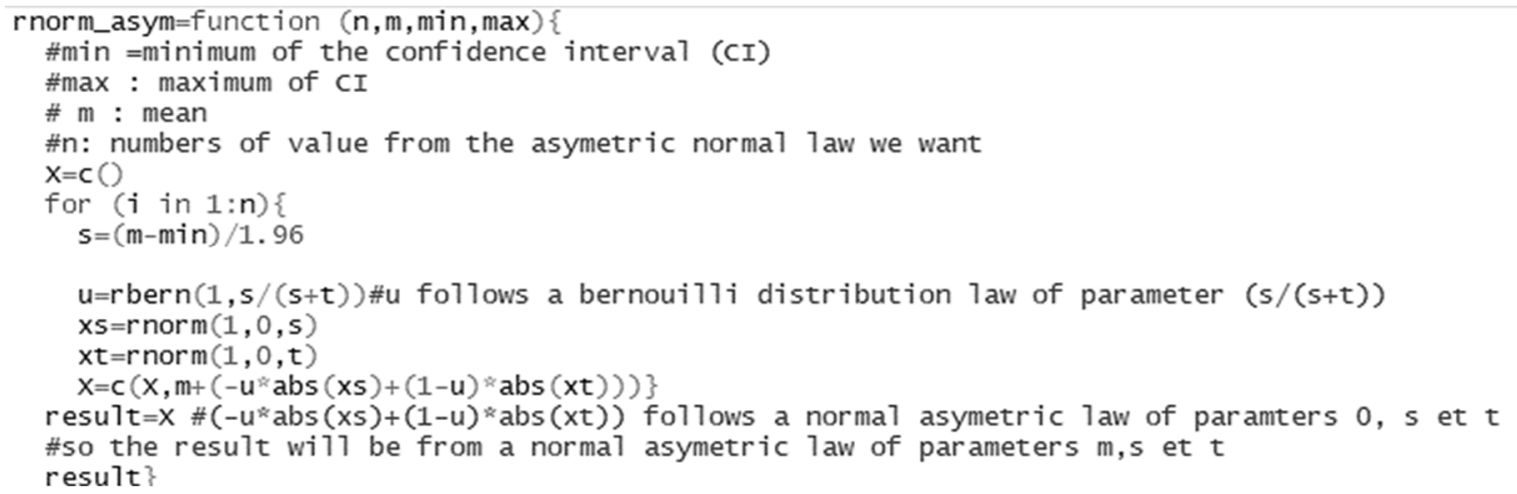

\section{Appendix 2. Calculation of the proportion of animals with detectable lesions by the infectious bTB status of the animal}

We used data for a French study (Courcoul et al., 2014) on the results of the diagnostic slaughters realised in an infected French department (Dordogne) between 2008 and 2012. Table A presents the presence of detectable lesions and the results by PCR and bacteriology of the 5,211 non-negative animals by ICT screening and slaughtered for diagnosis.

TableA: Presence of detectable lesions and results to PCR and bacteriology of slaughtered animals for diagnosis in Dordogne between 2008 and 2012 (Courcoul et al. 2014).

Confirmed infected $(\mathrm{C}+) \quad$ Unconfirmed $\quad$ Total

$(\mathbf{P C R}+$ and/or bacteriology +$)$

(C-)

$\begin{array}{llrl}\text { Detectable lesions (L+) } & 305(70.4 \%) & 392(8.2 \%) & 697\end{array}$




\begin{tabular}{cccc}
\hline No detectable lesions (L-) & $128(29.6 \%)$ & $4,386(91.8 \%)$ & 4,514 \\
\hline Total & $433(100 \%)$ & $4,778(100 \%)$ & 5,211 \\
\hline
\end{tabular}

We used these data and the characteristics of the PCR and bacteriology results to calculate the proportion of animals with detectable lesions among infected and uninfected animals to use as parameters in our scenario trees.

First, we estimated the proportion of infected animals in these data, $\mathrm{p}(\mathrm{I}+\mid \mathrm{L}+)$ and $\mathrm{p}(\mathrm{I}+\mid \mathrm{L}-)$ :

We had, according to (Gardner et al., 2000):

$\mathrm{Se}_{\mathrm{L}+}=1-\left(1-\mathrm{Se}_{\mathrm{PCR} \mid \mathrm{L}+}\right) *\left(1-\mathrm{Se}_{\text {culture } \mid \mathrm{L}+}\right)-\mathrm{Y}_{\mathrm{Se} \mid \mathrm{L}+}$, with $\mathrm{Se}_{\mathrm{L}+}$ the sensitivity of the use of PCR and bacteriology in parallel for animals with lesions, $\mathrm{Se}_{\mathrm{PCR} \mid \mathrm{L}+} \mathrm{PCR}$ sensitivity when there are lesions, $\mathrm{Se}_{\text {culture|L+ }}$ bacteriology sensitivity when there are lesions, and $\mathrm{Y}_{\mathrm{Se} \mid \mathrm{L}+}$ the covariance of sensitivity between these two tests when there are lesions in infected cattle.

$\mathrm{Sp}_{\mathrm{L}+}=\mathrm{Sp}_{\mathrm{PCR} \mid \mathrm{L}+} * \mathrm{Sp}_{\text {culture } \mid \mathrm{L}+}+\mathrm{Y}_{\mathrm{Sp} \mid \mathrm{L}+}$, with $\mathrm{Sp}_{\mathrm{L}+}$ the specificity of the use of PCR and bacteriology in parallel for animals with lesions, $\mathrm{Sp}_{\mathrm{PCR} \mid \mathrm{L}+} \mathrm{PCR}$ specificity when there are lesions, $\mathrm{Sp}_{\text {culture|L+ }}$ bacteriology specificity when there are lesions and $\mathrm{Y}_{\mathrm{Sp} \mid \mathrm{L}+}$ the covariance of specificity between these two tests when there are lesions in uninfected cattle.

$\mathrm{Se}_{\mathrm{L}-}=1-\left(1-\mathrm{Se}_{\mathrm{PCR} \mid \mathrm{L}-}\right) *\left(1-\mathrm{Se}_{\mathrm{cultur} \mid \mathrm{L}-}\right)-\mathrm{Y}_{\mathrm{Se} \mid \mathrm{L}-}$, with $\mathrm{Se}_{\mathrm{L}-}$ the sensitivity of the use of PCR and bacteriology in parallel for animals without lesions, $\mathrm{Se}_{\mathrm{PCR} \mid \mathrm{L}-} \mathrm{PCR}$ sensitivity without lesions, $\mathrm{Se}_{\text {culture|L- }}$ bacteriology

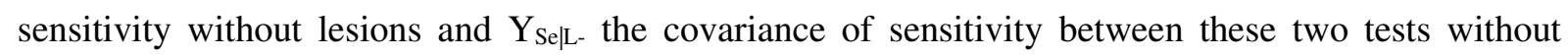
lesions in infected cattle.

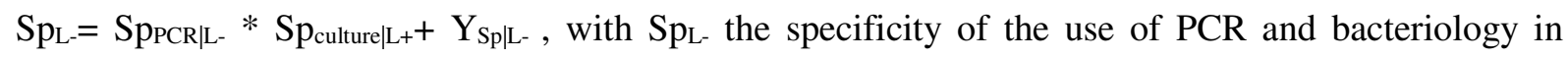
parallel for animals without lesions, $\mathrm{Sp}_{\mathrm{PCR} \mid \mathrm{L}-} \mathrm{PCR}$ specificity without lesions, $\mathrm{Sp}_{\text {culture|L- bacteriology }}$

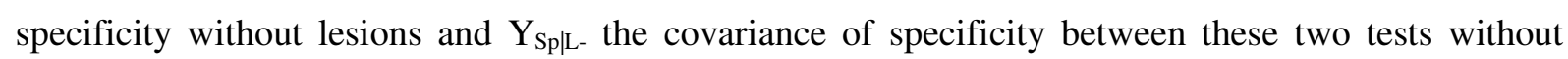
lesions in uninfected cattle.

And according to table A: 
$\mathrm{p}(\mathrm{C}+\mid \mathrm{L}+)=305 / 697=0.44$

$\mathrm{p}(\mathrm{C}+\mid \mathrm{L}-)=128 / 4,514=0.028$

$\mathrm{p}(\mathrm{L}+)=697 / 5,211=0.13$

$\mathrm{p}(\mathrm{L}-)=4,514 / 5,211=0.87$

Therefore,

$\mathrm{p}(\mathrm{I}+\mid \mathrm{L}+)=\left(\mathrm{p}(\mathrm{C}+\mid \mathrm{L}+)-\left(1-\mathrm{Sp}_{\mid \mathrm{L}+}\right)\right) /\left(\operatorname{Se}_{\mid \mathrm{L}+}+\mathrm{Sp}_{\mid \mathrm{L}+}-1\right)$ and $\mathrm{p}(\mathrm{I}+\mid \mathrm{L}-)=\left(\mathrm{p}(\mathrm{C}+\mid \mathrm{L}-)-\left(1-\mathrm{Sp}_{\mid \mathrm{L}-}\right)\right) /\left(\mathrm{Se}_{\mid \mathrm{L}-}+\mathrm{Sp}_{\mid \mathrm{L}-}-\right.$

1)

In addition, we can estimate disease prevalence in the sample :

$\mathrm{p}(\mathrm{I}+)=\mathrm{p}(\mathrm{I}+\mid \mathrm{L}+)^{*} \mathrm{p}(\mathrm{L}+)+\mathrm{p}(\mathrm{I}+\mid \mathrm{L}-)^{*} \mathrm{p}(\mathrm{L}-)$

$\mathrm{p}(\mathrm{I}+)=\left(\left(\mathrm{p}(\mathrm{C}+\mid \mathrm{L}+)-\left(1-\mathrm{Sp}_{\mid \mathrm{L}+}\right)\right) /\left(\mathrm{Se}_{\mid \mathrm{L}+}+\mathrm{Sp}_{\mid \mathrm{L}+}-1\right)\right) * 0.13+\left(\left(\mathrm{p}(\mathrm{C}+\mid \mathrm{L}-)-\left(1-\mathrm{Sp}_{\mid \mathrm{L}-}\right)\right) /\left(\mathrm{Se}_{\mid \mathrm{L}-}+\mathrm{Sp}_{\mid \mathrm{L}-}-1\right)\right) * 0.87$

$\mathrm{p}(\mathrm{I}-)=1-\mathrm{p}(\mathrm{I}+)$

From these equations and with Bayes theorem, we calculated for each model iteration the probability to have detectable lesions for an infected animal $(\mathrm{p}(\mathrm{L}+\mid \mathrm{I}+))$ and an uninfected one $(\mathrm{p}(\mathrm{L}+\mid \mathrm{I}-))$ :

$\mathbf{p}(\mathbf{L}+\mid \mathbf{I}+)=p(\mathrm{I}+\mid \mathrm{L}+)^{*} \mathrm{p}(\mathrm{L}+) / \mathrm{p}(\mathrm{I}+)$

$\mathbf{p}(\mathbf{L}+\mid \mathbf{I}-)=p(\mathrm{I}-\mid \mathrm{L}+)^{*} \mathrm{p}(\mathrm{L}+) / \mathrm{p}(\mathrm{I}-)$

Finally, for 10,000 iterations, the calculated proportion of animals with detectable lesions among infected and uninfected animals was 73\% (CI95\%: [71.3\%-76.1\%]) and 8.1\% (CI95\%: [7.6\%-8.5\%]), respectively.

Appendix 3. Sensitivity and specificity covariances of two dependent tests used in parallel (Gardner et al., 2000)

If we consider two tests of sensitivity, $\mathrm{Se} 1$ and $\mathrm{Se} 2$, and specificity, $\mathrm{Sp} 1$ and $\mathrm{Sp} 2$,

- the sensitivity covariance has to be:

$\circ$ greater than the maximum between $-(1-\mathrm{Se} 1)(1-\mathrm{Se} 2)$ and $-\mathrm{Se} 1 * \mathrm{Se} 2$

- and less than the minimum between $\mathrm{Se} 1 *(1-\mathrm{Se} 2)$ and $\mathrm{Se} 2 *(1-\mathrm{Se} 1)$

- the specificity covariance has to be: 
$\circ$ greater than the maximum between $-(1-\mathrm{Sp} 1)(1-\mathrm{Sp} 2)$ and $-\mathrm{Sp} 1 * \mathrm{Sp} 2$

$\circ$ and less than the minimum between $\mathrm{Sp} 1 *(1-\mathrm{Sp} 2)$ and $\mathrm{Sp} 2 *(1-\mathrm{Sp} 1)$

Appendix 4. Results of Buczinski and Reuillon, 2015(table A) the estimation of daily care costs by production type and type of animal and the method used to group some of these results into larger animal type categories

Table A: Result of Buczinski and Reuillon 2015 estimation of daily care cost in euros per day

\begin{tabular}{|c|c|c|c|}
\hline \multirow{3}{*}{\multicolumn{2}{|c|}{ Type of animal }} & \multicolumn{2}{|c|}{ Production type } \\
\hline & & \multicolumn{2}{|c|}{ Dairy } \\
\hline & & Plain dairy & Mountain dairy \\
\hline \multicolumn{2}{|c|}{ Female $\geq 24$ months } & 3.18 & 4.18 \\
\hline \multicolumn{2}{|c|}{ Female $<24$ months } & 2.87 & 3.79 \\
\hline \multicolumn{2}{|c|}{ Dairy male calf * } & 103.66 & 122.06 \\
\hline & & \multicolumn{2}{|c|}{ Beef } \\
\hline & & Beef & Beef / fattening \\
\hline \multicolumn{2}{|c|}{ Cow $\geq 24$ months } & 3.68 & 3.70 \\
\hline \multicolumn{2}{|c|}{ Pregnant 3-year old heifer } & 3.29 & 3.68 \\
\hline \multirow{2}{*}{$\begin{array}{c}\text { Female }<24 \\
\text { months }\end{array}$} & Heifer $\leq$ 1year old & 1.92 & 1.88 \\
\hline & Heifer > 1-year old & 2.39 & 2.33 \\
\hline \multicolumn{2}{|c|}{ Male $<6$ months } & 1.30 & 1.42 \\
\hline \multicolumn{2}{|c|}{ Male $\geq 6$ months } & 3.14 & 3.32 \\
\hline
\end{tabular}

For beef farms, we grouped the estimation for cows older than 24 months and pregnant three-year-old heifers into the category "female older than 24 months". We chose the parameters of the uniform law to describe the daily care costs of this group as the minimum and maximum value among the four estimated.

We also grouped the estimation for one and two-year-old heifers into the category "female younger than 24 months". An analyses of BDNI data showed that, in most French beef and beef/fattening farms, there are more heifers under one year of age than heifers between one and two years of age: the mean proportion of heifers under one year of age among heifers younger than two years of age was $64 \%$. We thus estimated the daily care cost of females under 24 months of age as the weighted mean of this cost for a one-year-old heifer and a two-year-old heifer with coefficients of 0.64 for one-yearolds and 0.36 for two-year-olds. We obtained a daily care cost of $2.09 € \mathrm{DF}$ for females under 24 months of age in beef farms and of $2.04 € \mathrm{DF}$ for the same category of animals in beef/fattening farms. 
Appendix 5: Mean (table A) and standard (table B) deviation of the normal distribution law best fitted to the number of each type of animal sold each month of 2016 in France for each type of herd (source: BDNI)

Legend: Fsup24: female older than 24 months, Finf24: female younger than 24 months, Msup6: male older than 6 months, Minf6: male younger than 6 months, Dairycalf:

dairy calves, Dssup: small dairy farm with a turnover of greater than $40 \%$, Dsinf: small dairy farm with a turnover of less than $40 \%$, Dbsup: big dairy farm with a turnover of greater than 40\%, Dbinf: big dairy farm with a turnover of less than $40 \%$, Bssup: small beef farm with a turnover of greater than $40 \%$, Bsinf: small beef farm with a turnover of less than $40 \%$, Bbsup: big beef farm with a turnover of greater than $40 \%$, Bbinf: big beef farm with a turnover of less than $40 \%$,

Mssup: small mixed farm with a turnover of greater than 40\%, Msinf: small mixed farm with a turnover of less than $40 \%$, Mbsup: big mixed farm with a turnover of greater than $40 \%$, Mbinf: big mixed farm with a turnover of less than $40 \%$.

Table A: Mean of the normal distribution law best fitted to the number of each type of animal sold each month of 2016 in France for each type of herd (data from the BDNI)

\begin{tabular}{|c|c|c|c|c|c|c|c|c|c|c|c|c|}
\hline \multirow{2}{*}{ Animal and herd type } & \multicolumn{12}{|c|}{ Month } \\
\hline & 1 & 2 & 3 & 4 & 5 & 6 & 7 & 8 & 9 & 10 & 11 & 12 \\
\hline Fsup24_Dssup & 2.02 & 3.63 & 4.72 & 1.58 & 7.32 & 2.75 & 17.22 & 14.31 & 3.39 & 20.10 & 0.00 & 0.00 \\
\hline Finf24_Dssup & 2.33 & 4.12 & 6.58 & 1.97 & 6.59 & 1.84 & 13.13 & 2.34 & 2.75 & 22.73 & 0.00 & 0.00 \\
\hline Msup6_Dssup & 2.00 & 4.59 & 1.56 & 2.62 & 7.82 & 2.61 & 9.56 & 2.75 & 17.22 & 14.31 & 0.00 & 0.00 \\
\hline Minf6_Dssup & 1.50 & 4.67 & 1.67 & 6.39 & 6.36 & 3.39 & 20.10 & 1.84 & 13.13 & 0.00 & 0.00 & 0.00 \\
\hline Dairycalf_Dssup & 2.40 & 3.80 & 1.93 & 5.38 & 2.34 & 2.75 & 22.73 & 2.61 & 9.56 & 0.00 & 0.00 & 0.00 \\
\hline Fsup24_Dsinf & 1.30 & 1.64 & 2.51 & 0.54 & 1.58 & 0.33 & 1.27 & 1.94 & 0.33 & 1.23 & 0.20 & 1.99 \\
\hline Finf24_Dsinf & 1.43 & 2.65 & 2.58 & 0.65 & 1.22 & 0.34 & 1.45 & 0.25 & 0.32 & 1.50 & 0.28 & 1.44 \\
\hline Msup6_Dsinf & 1.13 & 2.82 & 0.66 & 0.78 & 1.35 & 0.25 & 1.67 & 0.33 & 1.27 & 1.94 & 0.42 & 1.36 \\
\hline Minf6_Dsinf & 0.94 & 2.41 & 0.69 & 1.59 & 1.58 & 0.33 & 1.23 & 0.34 & 1.45 & 0.00 & 0.16 & 1.65 \\
\hline Dairycalf_Dsinf & 1.40 & 1.95 & 0.65 & 1.77 & 0.25 & 0.32 & 1.50 & 0.25 & 1.67 & 0.19 & 0.00 & 1.70 \\
\hline Fsup24_Dbsup & 5.01 & 6.50 & 8.36 & 2.84 & 12.17 & 7.25 & 14.30 & 23.38 & 7.61 & 20.12 & 0.00 & 0.00 \\
\hline
\end{tabular}




\begin{tabular}{|c|c|c|c|c|c|c|c|c|c|c|c|c|}
\hline \multirow{2}{*}{ Animal and herd type } & \multicolumn{12}{|c|}{ Month } \\
\hline & 1 & 2 & 3 & 4 & 5 & 6 & 7 & 8 & 9 & 10 & 11 & 12 \\
\hline Finf24_Dbsup & 5.68 & 9.24 & 10.32 & 2.41 & 8.24 & 8.46 & 20.73 & 5.97 & 8.76 & 21.32 & 0.00 & 0.00 \\
\hline Msup6_Dbsup & 4.79 & 10.27 & 2.79 & 4.12 & 5.03 & 6.91 & 22.34 & 7.25 & 14.30 & 23.38 & 0.00 & 0.00 \\
\hline Minf6_Dbsup & 4.16 & 8.85 & 2.78 & 8.67 & 9.76 & 7.61 & 20.12 & 8.46 & 20.73 & 0.00 & 0.00 & 0.00 \\
\hline Dairycalf_Dbsup & 5.12 & 8.44 & 4.15 & 7.67 & 5.97 & 8.76 & 21.32 & 6.91 & 22.34 & 0.00 & 0.00 & 0.00 \\
\hline Fsup24_Dbinf & 3.14 & 3.41 & 4.07 & 0.85 & 2.96 & 1.08 & 2.76 & 2.92 & 1.08 & 3.05 & 0.64 & 2.61 \\
\hline Finf24_Dbinf & 3.21 & 4.46 & 4.13 & 1.03 & 1.98 & 1.16 & 3.28 & 0.86 & 0.95 & 3.09 & 0.83 & 2.51 \\
\hline Msup6_Dbinf & 2.66 & 5.74 & 1.03 & 1.28 & 2.04 & 0.96 & 3.75 & 1.08 & 2.76 & 2.92 & 1.08 & 2.34 \\
\hline Minf6_Dbinf & 2.14 & 5.13 & 1.05 & 2.20 & 2.45 & 1.08 & 3.05 & 1.16 & 3.28 & 0.06 & 0.51 & 2.79 \\
\hline Dairycalf_Dbinf & 2.95 & 3.47 & 1.02 & 3.24 & 0.86 & 0.95 & 3.09 & 0.96 & 3.75 & 0.50 & 2.45 & 2.81 \\
\hline Fsup24_Bssup & 1.75 & 3.00 & 5.50 & 1.49 & 7.91 & 4.42 & 19.84 & 17.08 & 4.71 & 21.92 & 0.00 & 0.00 \\
\hline Finf24_Bssup & 1.74 & 4.09 & 6.15 & 1.87 & 6.45 & 4.96 & 19.37 & 4.39 & 5.00 & 20.27 & 0.00 & 0.00 \\
\hline Msup6_Bssup & 2.31 & 4.09 & 1.55 & 3.04 & 9.03 & 4.15 & 21.74 & 4.42 & 19.84 & 17.08 & 0.00 & 0.00 \\
\hline Minf6_Bssup & 2.04 & 5.54 & 1.80 & 7.17 & 10.64 & 4.71 & 21.92 & 4.96 & 19.37 & 0.00 & 0.00 & 0.00 \\
\hline Dairycalf_Bssup & 2.41 & 5.00 & 2.09 & 6.46 & 4.39 & 5.00 & 20.27 & 4.15 & 21.74 & 0.00 & 0.00 & 0.00 \\
\hline Fsup24_Bsinf & 0.67 & 0.94 & 1.87 & 0.41 & 1.87 & 0.91 & 2.36 & 2.90 & 1.05 & 1.88 & 0.00 & 0.00 \\
\hline Finf24_Bsinf & 0.78 & 2.03 & 2.48 & 0.62 & 1.26 & 0.89 & 2.29 & 1.04 & 1.44 & 2.51 & 0.00 & 0.00 \\
\hline Msup6_Bsinf & 0.77 & 2.15 & 0.66 & 0.90 & 1.73 & 0.64 & 2.26 & 0.91 & 2.36 & 2.90 & 0.00 & 0.00 \\
\hline Minf6_Bsinf & 0.49 & 2.05 & 0.77 & 1.84 & 2.19 & 1.05 & 1.88 & 0.89 & 2.29 & 0.00 & 0.00 & 0.00 \\
\hline Dairycalf_Bsinf & 0.66 & 1.42 & 0.71 & 1.95 & 1.04 & 1.44 & 2.51 & 0.64 & 2.26 & 0.00 & 0.00 & 0.00 \\
\hline Fsup24_Bbsup & 10.84 & 11.50 & 20.62 & 3.29 & 11.95 & 11.09 & 32.08 & 37.44 & 12.84 & 22.33 & 0.00 & 0.00 \\
\hline Finf24_Bbsup & 11.32 & 19.47 & 20.80 & 4.67 & 9.25 & 12.58 & 26.63 & 11.01 & 13.40 & 29.50 & 0.00 & 0.00 \\
\hline Msup6_Bbsup & 14.46 & 20.08 & 4.10 & 4.39 & 13.11 & 9.85 & 33.09 & 11.09 & 32.08 & 37.44 & 0.00 & 0.00 \\
\hline Minf6_Bbsup & 12.47 & 26.86 & 3.77 & 11.98 & 9.64 & 12.84 & 22.33 & 12.58 & 26.63 & 0.00 & 0.00 & 0.00 \\
\hline Dairycalf_Bbsup & 11.06 & 23.14 & 4.95 & 9.97 & 11.01 & 13.40 & 29.50 & 9.85 & 33.09 & 0.00 & 0.00 & 0.00 \\
\hline
\end{tabular}




\begin{tabular}{|c|c|c|c|c|c|c|c|c|c|c|c|c|}
\hline \multirow{2}{*}{ Animal and herd type } & \multicolumn{12}{|c|}{ Month } \\
\hline & 1 & 2 & 3 & 4 & 5 & 6 & 7 & 8 & 9 & 10 & 11 & 12 \\
\hline Fsup24_Bbinf & 2.13 & 2.46 & 3.75 & 1.01 & 4.30 & 3.36 & 5.92 & 6.31 & 3.87 & 5.37 & 0.00 & 0.00 \\
\hline Finf24_Bbinf & 2.62 & 3.93 & 4.50 & 1.57 & 2.79 & 3.31 & 5.80 & 3.41 & 4.06 & 6.47 & 0.00 & 0.00 \\
\hline Msup6_Bbinf & 2.90 & 4.68 & 1.53 & 1.95 & 3.66 & 2.61 & 5.88 & 3.36 & 5.92 & 6.31 & 0.00 & 0.00 \\
\hline Minf6_Bbinf & 1.88 & 5.44 & 1.95 & 3.56 & 4.11 & 3.87 & 5.37 & 3.31 & 5.80 & 0.00 & 0.00 & 0.00 \\
\hline Dairycalf_Bbinf & 2.15 & 3.37 & 1.85 & 4.18 & 3.41 & 4.06 & 6.47 & 2.61 & 5.88 & 0.00 & 0.00 & 0.00 \\
\hline Fsup24_Mssup & 2.99 & 4.69 & 7.58 & 2.61 & 6.24 & 4.86 & 13.68 & 8.32 & 5.89 & 29.59 & 0.00 & 0.00 \\
\hline Finf24_Mssup & 4.55 & 4.61 & 8.05 & 3.21 & 8.85 & 4.73 & 11.95 & 5.15 & 5.11 & 19.24 & 0.00 & 0.00 \\
\hline Msup6_Mssup & 3.87 & 9.42 & 2.38 & 2.07 & 9.41 & 6.50 & 12.37 & 4.86 & 13.68 & 8.32 & 0.00 & 0.00 \\
\hline Minf6_Mssup & 3.59 & 6.72 & 3.46 & 6.33 & 2.75 & 5.89 & 29.59 & 4.73 & 11.95 & 0.00 & 0.00 & 0.00 \\
\hline Dairycalf_Mssup & 4.02 & 7.88 & 2.56 & 8.51 & 5.15 & 5.11 & 19.24 & 6.50 & 12.37 & 0.00 & 0.00 & 0.00 \\
\hline Fsup24_Msinf & 2.01 & 2.41 & 3.26 & 0.83 & 2.22 & 1.39 & 2.86 & 3.29 & 1.52 & 2.64 & 0.00 & 1.71 \\
\hline Finf24_Msinf & 2.11 & 3.07 & 3.33 & 1.08 & 1.44 & 1.51 & 3.03 & 1.34 & 1.82 & 3.03 & 0.16 & 0.00 \\
\hline Msup6_Msinf & 1.99 & 3.02 & 1.07 & 1.36 & 1.88 & 1.14 & 3.74 & 1.39 & 2.86 & 3.29 & 0.22 & 1.54 \\
\hline Minf6_Msinf & 1.49 & 3.34 & 1.21 & 2.01 & 2.25 & 1.52 & 2.64 & 1.51 & 3.03 & 0.00 & 0.00 & 1.95 \\
\hline Dairycalf_Msinf & 2.00 & 2.40 & 1.20 & 2.05 & 1.34 & 1.82 & 3.03 & 1.14 & 3.74 & 0.04 & 0.00 & 0.00 \\
\hline Fsup24_Mbsup & 15.85 & 15.11 & 31.38 & 4.56 & 15.42 & 14.71 & 13.85 & 52.24 & 13.56 & 16.62 & 0.00 & 0.00 \\
\hline Finf24_Mbsup & 14.54 & 24.20 & 31.29 & 3.53 & 6.42 & 12.88 & 45.05 & 9.00 & 19.31 & 27.77 & 0.00 & 0.00 \\
\hline Msup6_Mbsup & 14.29 & 21.66 & 5.53 & 5.93 & 7.05 & 10.53 & 22.60 & 14.71 & 13.85 & 52.24 & 0.00 & 0.00 \\
\hline Minf6_Mbsup & 16.01 & 20.88 & 4.10 & 12.56 & 14.68 & 13.56 & 16.62 & 12.88 & 45.05 & 0.00 & 0.00 & 0.00 \\
\hline Dairycalf_Mbsup & 16.71 & 25.60 & 6.48 & 6.93 & 9.00 & 19.31 & 27.77 & 10.53 & 22.60 & 0.00 & 0.00 & 0.00 \\
\hline Fsup24_Mbinf & 4.54 & 4.81 & 4.56 & 1.22 & 3.43 & 3.60 & 5.40 & 6.23 & 3.71 & 5.47 & 0.28 & 2.70 \\
\hline Finf24_Mbinf & 4.78 & 4.56 & 5.34 & 1.59 & 2.43 & 3.88 & 6.01 & 3.21 & 3.81 & 5.80 & 0.46 & 2.66 \\
\hline Msup6_Mbinf & 4.34 & 5.70 & 1.54 & 1.92 & 2.68 & 3.29 & 6.17 & 3.60 & 5.40 & 6.23 & 0.78 & 2.28 \\
\hline Minf6_Mbinf & 3.46 & 4.98 & 1.81 & 2.66 & 3.06 & 3.71 & 5.47 & 3.88 & 6.01 & 0.00 & 0.23 & 2.75 \\
\hline
\end{tabular}




\begin{tabular}{|c|c|c|c|c|c|c|c|c|c|c|c|c|}
\hline \multirow{2}{*}{ Animal and herd type } & \multicolumn{12}{|c|}{ Month } \\
\hline & $\mathbf{1}$ & 2 & 3 & 4 & 5 & 6 & 7 & 8 & 9 & 10 & 11 & 12 \\
\hline Dairycalf Mbinf & 4.11 & 4.02 & 1.73 & 3.75 & 3.21 & 3.81 & 5.80 & 3.29 & 6.17 & 0.15 & 0.00 & 2.70 \\
\hline
\end{tabular}

Table B. Standard deviation of the normal distribution law best fitted to the number of each type of animal sold each month of 2016 in France for each type of herd (from BDNI)

\begin{tabular}{|c|c|c|c|c|c|c|c|c|c|c|c|c|}
\hline \multirow{2}{*}{ Animal and herd type } & \multicolumn{12}{|c|}{ Month } \\
\hline & 1 & 2 & 3 & 4 & 5 & 6 & 7 & 8 & 9 & 10 & 11 & 12 \\
\hline Fsup24_Dssup & 1.89 & 2.71 & 4.94 & 1.58 & 6.02 & 2.24 & 12.41 & 12.27 & 3.98 & 18.12 & 0.00 & 0.00 \\
\hline Finf24_Dssup & 1.90 & 3.78 & 6.61 & 2.07 & 5.03 & 3.11 & 12.03 & 2.20 & 2.39 & 21.30 & 0.00 & 0.00 \\
\hline Msup6_Dssup & 1.94 & 4.03 & 1.33 & 2.03 & 6.28 & 3.33 & 19.19 & 2.24 & 12.41 & 12.27 & 0.00 & 0.00 \\
\hline Minf6_Dssup & 2.21 & 4.34 & 1.68 & 3.80 & 7.85 & 3.98 & 18.12 & 3.11 & 12.03 & 0.00 & 0.00 & 0.00 \\
\hline Dairycalf_Dssup & 2.68 & 5.03 & 1.64 & 5.41 & 2.20 & 2.39 & 21.30 & 3.33 & 19.19 & 0.00 & 0.00 & 0.00 \\
\hline Fsup24_Dsinf & 1.29 & 1.43 & 2.47 & 0.63 & 1.31 & 0.35 & 1.33 & 1.16 & 0.30 & 1.46 & 0.27 & 1.43 \\
\hline Finf24_Dsinf & 1.29 & 2.36 & 2.99 & 0.71 & 1.23 & 0.32 & 1.95 & 0.27 & 0.23 & 1.37 & 0.32 & 1.42 \\
\hline Msup6_Dsinf & 1.11 & 2.71 & 0.63 & 0.71 & 1.51 & 0.32 & 1.64 & 0.35 & 1.33 & 1.16 & 0.32 & 1.60 \\
\hline Minf6_Dsinf & 1.26 & 2.27 & 0.69 & 1.37 & 1.64 & 0.30 & 1.46 & 0.32 & 1.95 & 0.13 & 0.20 & 1.70 \\
\hline Dairycalf_Dsinf & 1.46 & 2.33 & 0.56 & 1.87 & 0.27 & 0.23 & 1.37 & 0.32 & 1.64 & 0.13 & 1.48 & 1.41 \\
\hline Fsup24_Dbsup & 5.07 & 5.85 & 8.01 & 2.78 & 10.97 & 7.57 & 15.09 & 18.65 & 7.30 & 18.00 & 0.00 & 0.00 \\
\hline Finf24_Dbsup & 4.97 & 7.82 & 10.68 & 3.93 & 6.88 & 7.53 & 23.69 & 6.00 & 6.47 & 20.10 & 0.00 & 0.00 \\
\hline Msup6_Dbsup & 4.75 & 9.12 & 2.54 & 3.90 & 9.89 & 7.60 & 26.89 & 7.57 & 15.09 & 18.65 & 0.00 & 0.00 \\
\hline Minf6_Dbsup & 4.66 & 9.22 & 3.29 & 5.95 & 13.07 & 7.30 & 18.00 & 7.53 & 23.69 & 0.00 & 0.00 & 0.00 \\
\hline Dairycalf_Dbsup & 5.33 & 8.80 & 3.22 & 7.86 & 6.00 & 6.47 & 20.10 & 7.60 & 26.89 & 0.00 & 0.00 & 0.00 \\
\hline Fsup24_Dbinf & 2.97 & 3.07 & 4.39 & 0.99 & 2.60 & 1.10 & 3.39 & 2.91 & 1.01 & 3.39 & 0.77 & 2.33 \\
\hline Finf24_Dbinf & 2.87 & 3.94 & 4.43 & 1.13 & 1.69 & 1.12 & 3.84 & 0.92 & 0.79 & 3.19 & 0.86 & 2.35 \\
\hline Msup6_Dbinf & 2.62 & 5.57 & 0.97 & 1.21 & 2.41 & 1.16 & 3.31 & 1.10 & 3.39 & 2.91 & 0.85 & 2.67 \\
\hline
\end{tabular}




\begin{tabular}{|c|c|c|c|c|c|c|c|c|c|c|c|c|}
\hline Minf6_Dbinf & 2.77 & 4.55 & 1.06 & 1.98 & 2.47 & 1.01 & 3.39 & 1.12 & 3.84 & 0.41 & 0.55 & 2.82 \\
\hline Dairycalf_Dbinf & 3.01 & 3.80 & 0.91 & 3.63 & 0.92 & 0.79 & 3.19 & 1.16 & 3.31 & 0.44 & 2.52 & 2.55 \\
\hline Fsup24_Bssup & 1.69 & 2.59 & 6.58 & 1.85 & 7.34 & 4.32 & 14.87 & 19.19 & 5.85 & 21.52 & 0.00 & 0.00 \\
\hline Finf24_Bssup & 1.95 & 3.98 & 6.11 & 2.50 & 7.78 & 4.93 & 19.19 & 3.68 & 4.62 & 29.48 & 0.00 & 0.00 \\
\hline Msup6_Bssup & 1.91 & 4.58 & 1.49 & 2.17 & 9.43 & 5.44 & 22.18 & 4.32 & 14.87 & 19.19 & 0.00 & 0.00 \\
\hline Minf6_Bssup & 2.88 & 5.02 & 2.09 & 5.59 & 8.12 & 5.85 & 21.52 & 4.93 & 19.19 & 0.00 & 0.00 & 0.00 \\
\hline Dairycalf_Bssup & 2.73 & 7.55 & 1.63 & 8.27 & 3.68 & 4.62 & 29.48 & 5.44 & 22.18 & 0.00 & 0.00 & 0.00 \\
\hline Fsup24_Bsinf & 0.66 & 0.82 & 2.11 & 0.46 & 1.53 & 0.90 & 2.28 & 2.30 & 1.26 & 2.30 & 0.00 & 0.00 \\
\hline Finf24_Bsinf & 0.82 & 1.91 & 2.48 & 0.73 & 1.43 & 0.82 & 2.31 & 0.99 & 0.94 & 2.80 & 0.00 & 0.00 \\
\hline Msup6_Bsinf & 0.58 & 2.27 & 0.70 & 0.67 & 1.95 & 0.84 & 2.40 & 0.90 & 2.28 & 2.30 & 0.00 & 0.00 \\
\hline Minf6_Bsinf & 0.59 & 1.52 & 0.83 & 1.82 & 1.91 & 1.26 & 2.30 & 0.82 & 2.31 & 0.00 & 0.00 & 0.00 \\
\hline Dairycalf_Bsinf & 0.75 & 1.66 & 0.52 & 2.09 & 0.99 & 0.94 & 2.80 & 0.84 & 2.40 & 0.00 & 0.00 & 0.00 \\
\hline Fsup24_Bbsup & 10.85 & 13.10 & 21.50 & 3.98 & 12.22 & 10.81 & 41.19 & 24.72 & 11.98 & 24.11 & 0.00 & 0.00 \\
\hline Finf24_Bbsup & 12.73 & 21.41 & 25.83 & 3.67 & 10.08 & 11.59 & 29.97 & 12.14 & 10.12 & 28.43 & 0.00 & 0.00 \\
\hline Msup6_Bbsup & 11.18 & 24.09 & 4.34 & 3.84 & 7.92 & 11.83 & 29.51 & 10.81 & 41.19 & 24.72 & 0.00 & 0.00 \\
\hline Minf6_Bbsup & 13.75 & 20.75 & 5.36 & 14.05 & 11.90 & 11.98 & 24.11 & 11.59 & 29.97 & 0.00 & 0.00 & 0.00 \\
\hline Dairycalf_Bbsup & 11.57 & 25.71 & 4.00 & 11.24 & 12.14 & 10.12 & 28.43 & 11.83 & 29.51 & 0.00 & 0.00 & 0.00 \\
\hline Fsup24_Bbinf & 2.21 & 2.32 & 3.99 & 1.26 & 3.33 & 3.18 & 5.80 & 5.30 & 4.03 & 6.33 & 0.00 & 0.00 \\
\hline Finf24_Bbinf & 2.69 & 4.68 & 4.72 & 1.69 & 3.23 & 3.22 & 5.89 & 3.32 & 2.92 & 6.46 & 0.00 & 0.00 \\
\hline Msup6_Bbinf & 2.24 & 5.28 & 1.64 & 1.53 & 3.80 & 3.53 & 6.18 & 3.18 & 5.80 & 5.30 & 0.00 & 0.00 \\
\hline Minf6_Bbinf & 2.26 & 3.63 & 2.11 & 3.92 & 3.74 & 4.03 & 6.33 & 3.22 & 5.89 & 0.00 & 0.00 & 0.00 \\
\hline Dairycalf_Bbinf & 2.27 & 3.77 & 1.31 & 4.61 & 3.32 & 2.92 & 6.46 & 3.53 & 6.18 & 0.00 & 0.00 & 0.00 \\
\hline Fsup24_Mssup & 3.47 & 4.26 & 8.37 & 2.02 & 4.91 & 3.59 & 8.01 & 15.32 & 6.74 & 14.63 & 0.00 & 0.00 \\
\hline Finf24_Mssup & 4.13 & 5.72 & 7.31 & 2.39 & 5.86 & 6.60 & 9.46 & 3.56 & 5.55 & 19.43 & 0.00 & 0.00 \\
\hline Msup6_Mssup & 3.76 & 6.97 & 2.08 & 2.14 & 4.62 & 5.81 & 24.06 & 3.59 & 8.01 & 15.32 & 0.00 & 0.00 \\
\hline Minf6_Mssup & 4.81 & 6.37 & 2.35 & 4.19 & 4.64 & 6.74 & 14.63 & 6.60 & 9.46 & 0.00 & 0.00 & 0.00 \\
\hline Dairycalf_Mssup & 4.48 & 10.00 & 2.11 & 4.65 & 3.56 & 5.55 & 19.43 & 5.81 & 24.06 & 0.00 & 0.00 & 0.00 \\
\hline Fsup24_Msinf & 1.99 & 2.12 & 2.76 & 0.98 & 1.91 & 1.41 & 2.89 & 2.85 & 1.65 & 3.06 & 0.13 & 0.00 \\
\hline
\end{tabular}




\begin{tabular}{|c|c|c|c|c|c|c|c|c|c|c|c|c|}
\hline Finf24_Msinf & 2.08 & 2.91 & 3.17 & 1.22 & 1.63 & 1.41 & 3.04 & 1.40 & 1.27 & 3.20 & 0.15 & 1.60 \\
\hline Msup6_Msinf & 1.78 & 3.52 & 1.16 & 1.14 & 2.01 & 1.43 & 2.95 & 1.41 & 2.89 & 2.85 & 0.13 & 1.79 \\
\hline Minf6_Msinf & 1.84 & 2.86 & 1.29 & 1.92 & 1.96 & 1.65 & 3.06 & 1.41 & 3.04 & 0.00 & 0.08 & 1.90 \\
\hline Dairycalf_Msinf & 2.03 & 2.65 & 0.96 & 2.41 & 1.40 & 1.27 & 3.20 & 1.43 & 2.95 & 0.00 & 0.00 & 1.63 \\
\hline Fsup24_Mbsup & 13.27 & 18.58 & 32.32 & 4.42 & 3.81 & 15.38 & 20.87 & 21.36 & 13.26 & 23.53 & 0.00 & 0.00 \\
\hline Finf24_Mbsup & 13.71 & 18.14 & 35.23 & 3.78 & 6.27 & 10.45 & 49.08 & 11.70 & 11.02 & 43.00 & 0.00 & 0.00 \\
\hline Msup6_Mbsup & 12.36 & 22.28 & 5.03 & 3.15 & 6.37 & 14.62 & 16.06 & 15.38 & 20.87 & 21.36 & 0.12 & 0.00 \\
\hline Minf6_Mbsup & 22.75 & 14.16 & 4.93 & 15.27 & 6.24 & 13.26 & 23.53 & 10.45 & 49.08 & 0.00 & 0.00 & 2.87 \\
\hline Dairycalf_Mbsup & 19.38 & 40.56 & 2.90 & 8.09 & 11.70 & 11.02 & 43.00 & 14.62 & 16.06 & 0.00 & 0.00 & 0.00 \\
\hline Fsup24_Mbinf & 4.44 & 4.46 & 5.50 & 1.45 & 2.76 & 3.46 & 5.69 & 5.39 & 3.48 & 6.09 & 0.45 & 2.30 \\
\hline Finf24_Mbinf & 4.19 & 6.13 & 5.47 & 1.70 & 3.03 & 3.71 & 5.96 & 3.41 & 3.02 & 5.60 & 0.60 & 2.33 \\
\hline Msup6_Mbinf & 3.95 & 5.69 & 1.58 & 1.78 & 2.99 & 3.86 & 5.92 & 3.46 & 5.69 & 5.39 & 0.49 & 2.76 \\
\hline Minf6_Mbinf & 4.33 & 4.31 & 1.89 & 3.35 & 3.19 & 3.48 & 6.09 & 3.71 & 5.96 & 0.02 & 0.30 & 2.69 \\
\hline Dairycalf_Mbinf & 4.22 & 4.76 & 1.39 & 4.27 & 3.41 & 3.02 & 5.60 & 3.86 & 5.92 & 0.19 & 2.32 & 2.35 \\
\hline
\end{tabular}




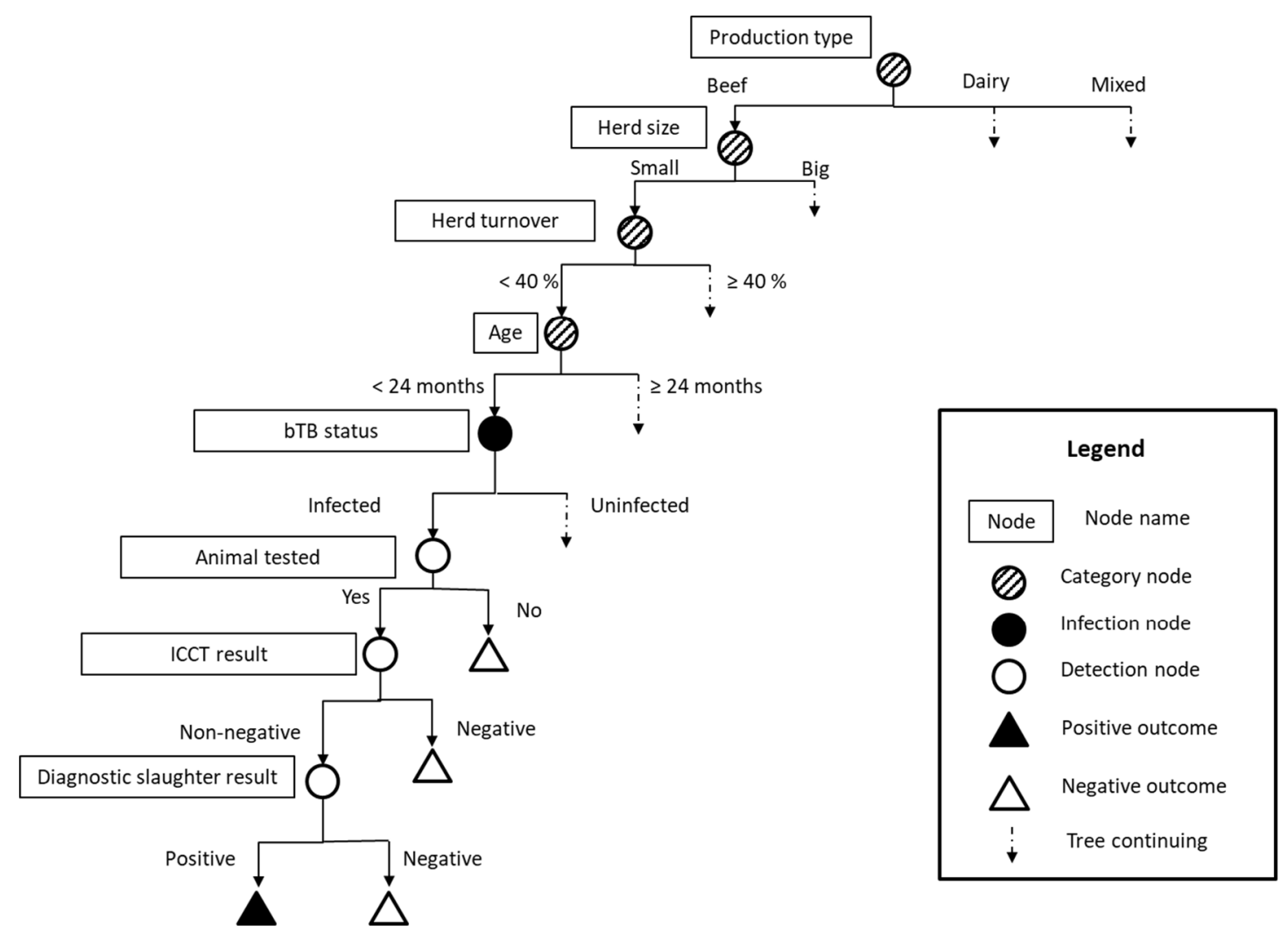




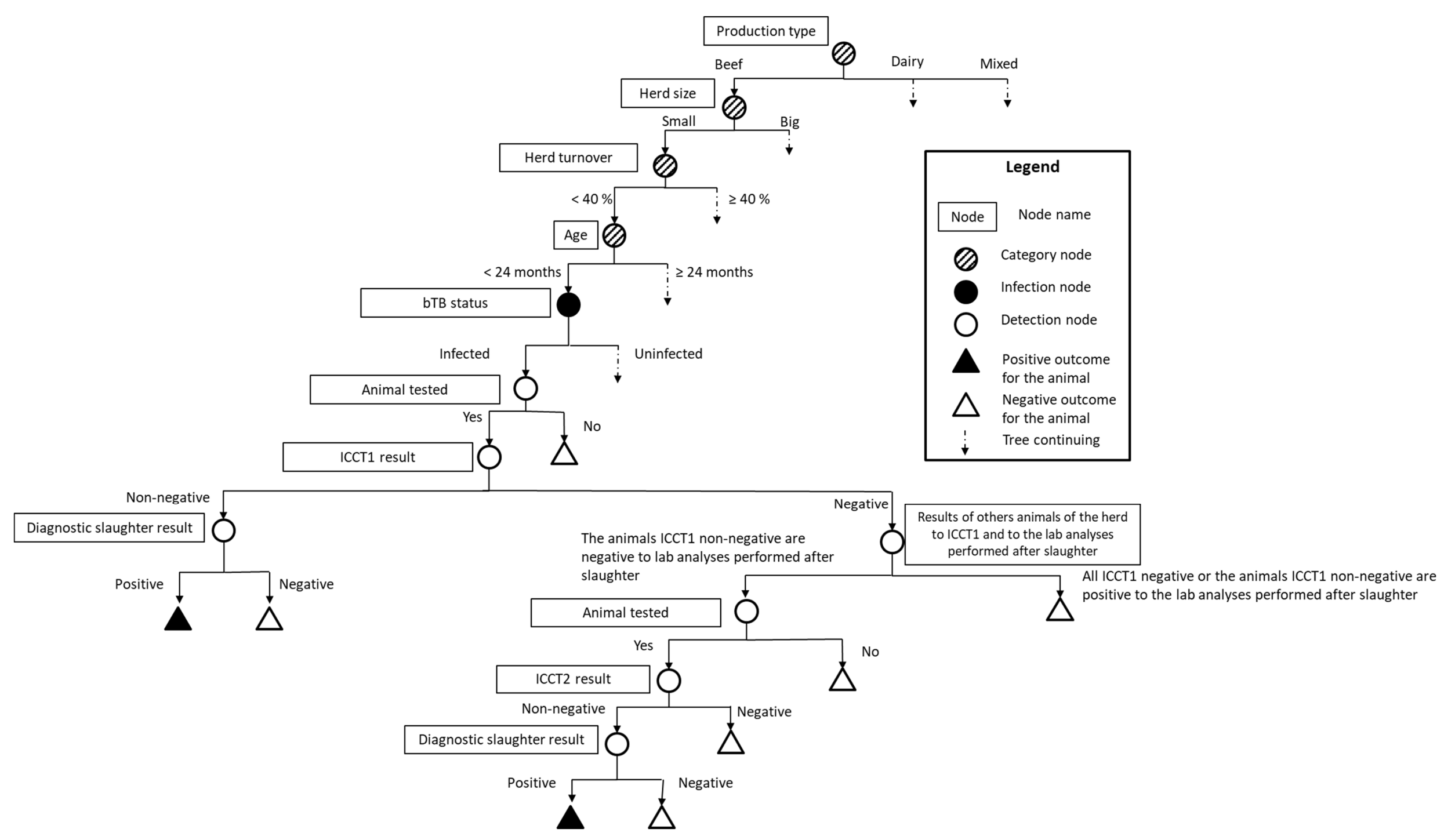




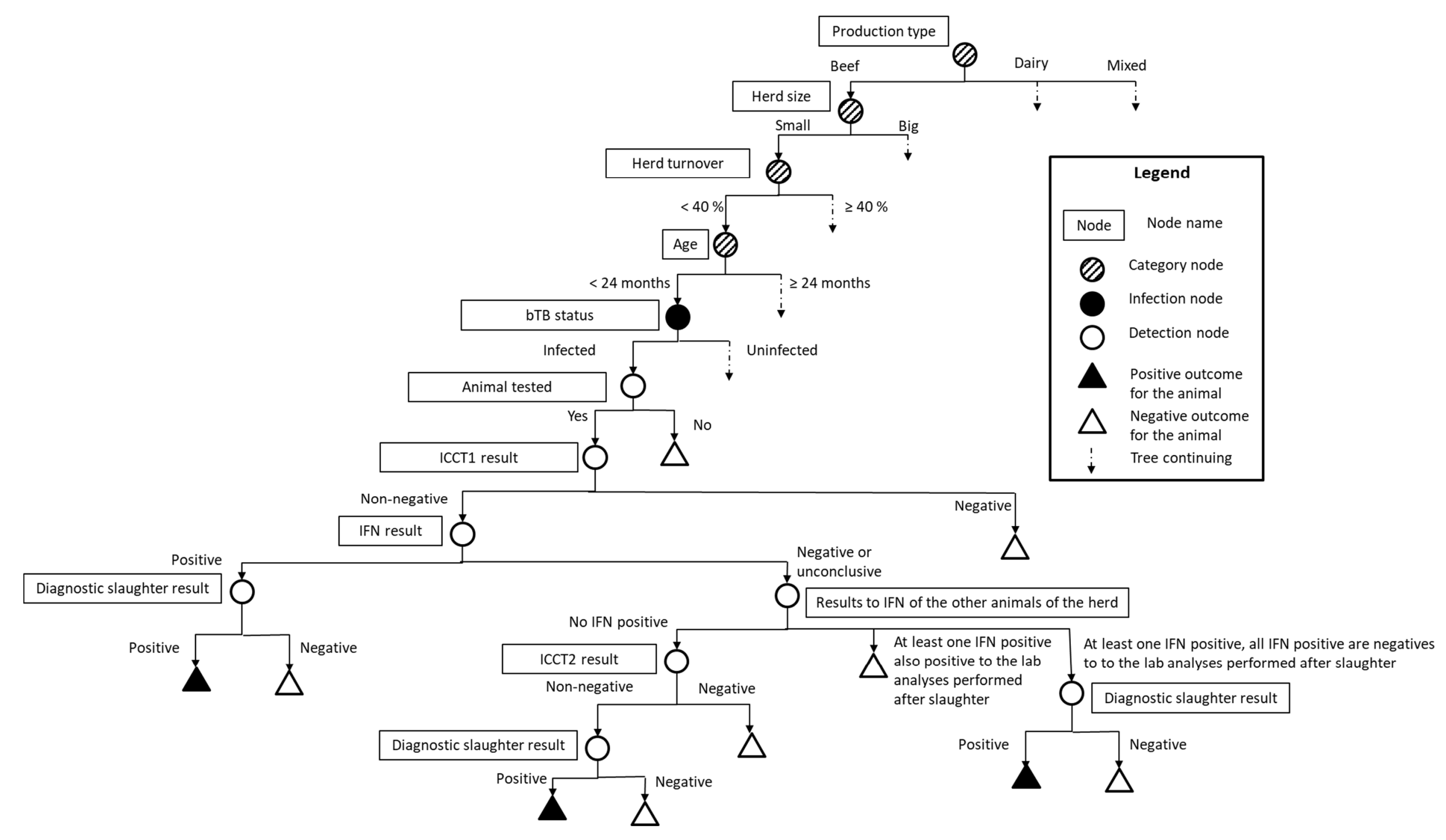




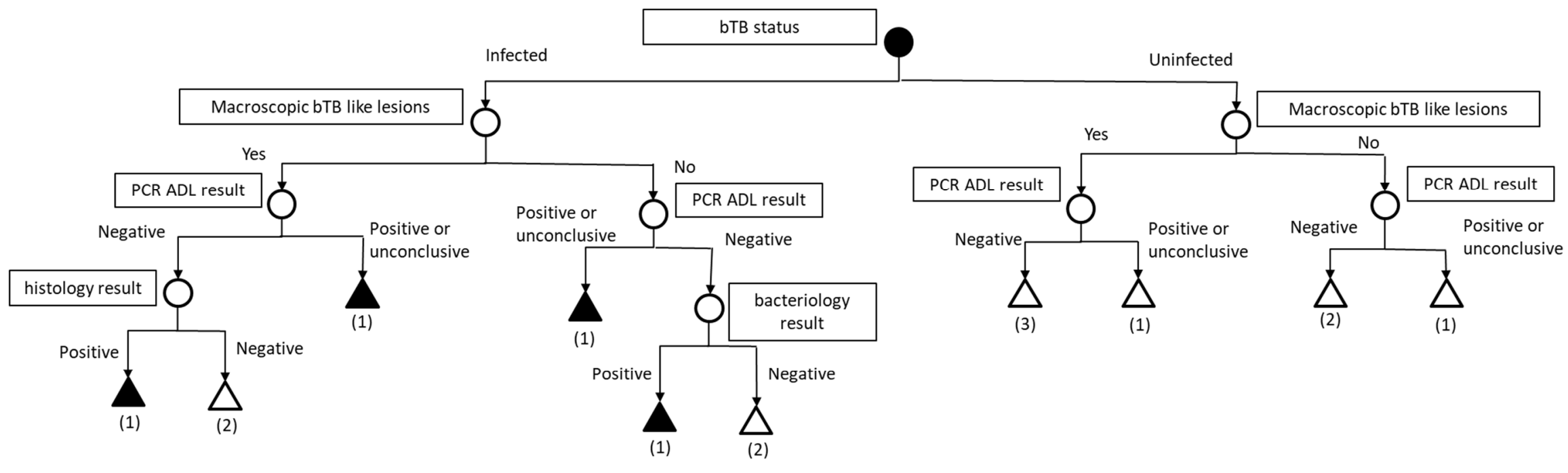

\begin{tabular}{l}
\hline Node Node name \\
(1) Samples are sent to the NRL for confirmation. The additionnal NRL confirmation methods (PCR, bacteriology, VNTR and spoligotypage) are considered to provide 100\% sensitivity and specificity. \\
(2) Sample is not sent to the NRL and no further analyses are conducted \\
(3) Histology is not represented because given the hypothesis the outcome will be the same for any histology result: if the histology is negative, the sample will not be sent to the NRL and the \\
diagnostic slaughter result will be considered to be negative; if it is positive, a sample will be sent to the NRL for confirmation and the NRL will refute this positive result as NRL is supposed to be \\
$100 \%$ specific
\end{tabular}




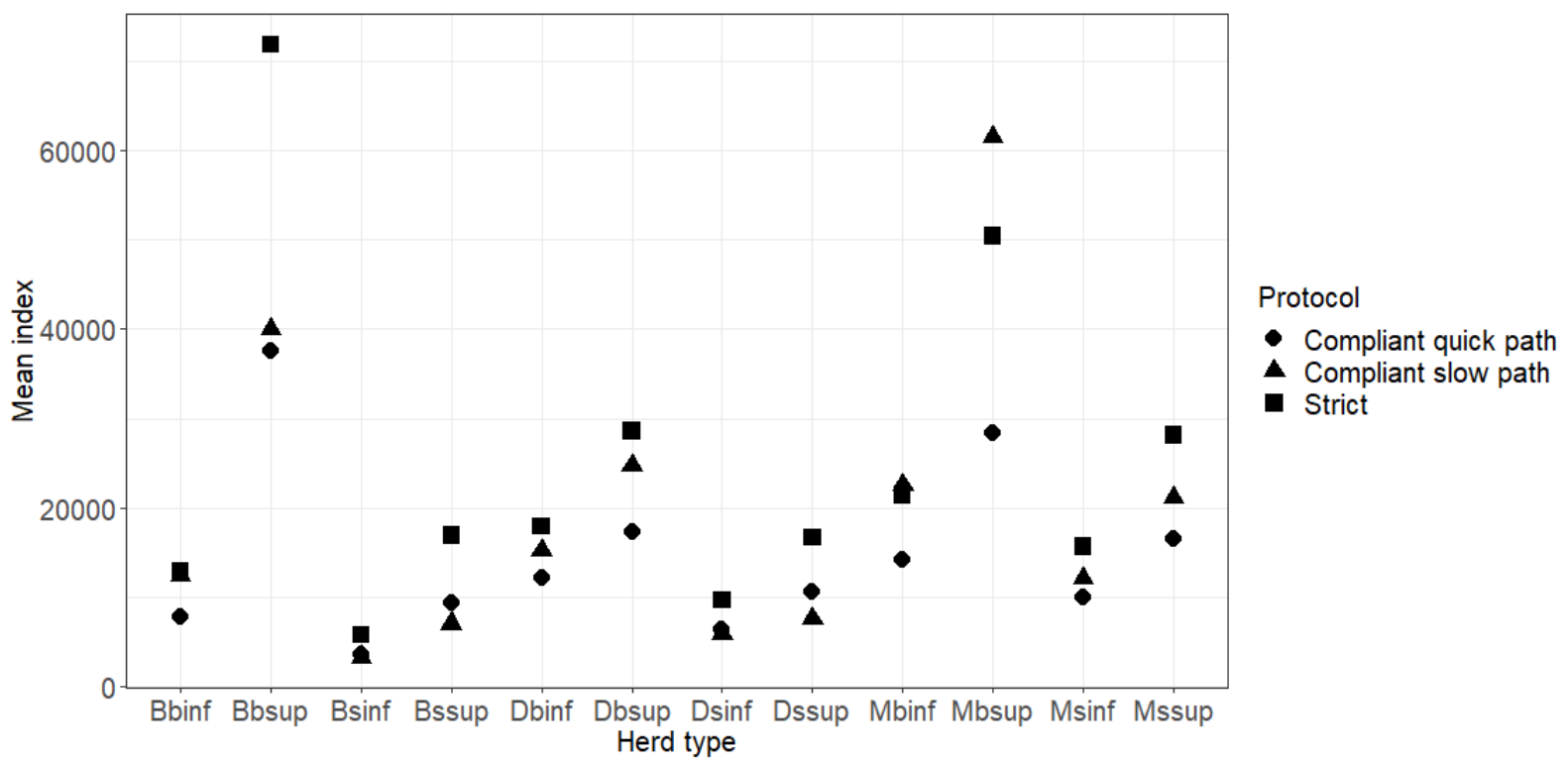

Legend: Bbinf: big beef farm with a turnover of less than $40 \%$, Bbsup: big beef farm with a turnover of greater than $40 \%$, Bsinf: small beef farm with a turnover of less than 40\%, Bssup: small beef farm with a turnover of greater than $40 \%$, Dbinf: big dairy farm with a turnover of less than 40\%, Dbsup: big dairy farm with a turnover of greater than 40\%, Dsinf: small dairy farm with a turnover of less than $40 \%$, Dssup: small dairy farm with a turnover of greater than $40 \%$, Mbinf: big mixed farm with a turnover of less than $40 \%$, Mbsup: big mixed farm with a turnover of greater than $40 \%$, Msinf: small mixed farm with a turnover of less than $40 \%$, Mssup: small mixed farm with a turnover of greater than $40 \%$.

A high cost-sensitivity index matches bad efficiency of the protocol. 


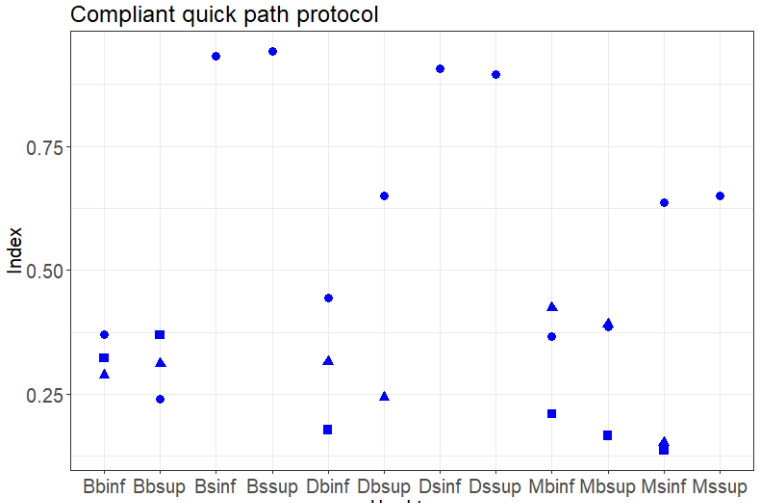

Bbinf Bbsup Bsinf Bssup Dbinf Dbsup Dsinf Dssup Mbinf Mbsup Msinf Mssup Herd type

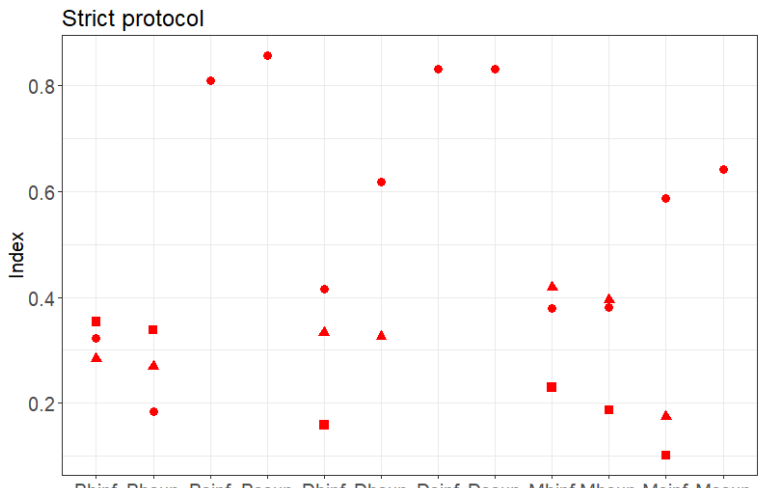

Bbinf Bbsup Bsinf Bssup Dbinf Dbsup Dsinf Dssup Mbinf Mbsup Msinf Mssup Herd type

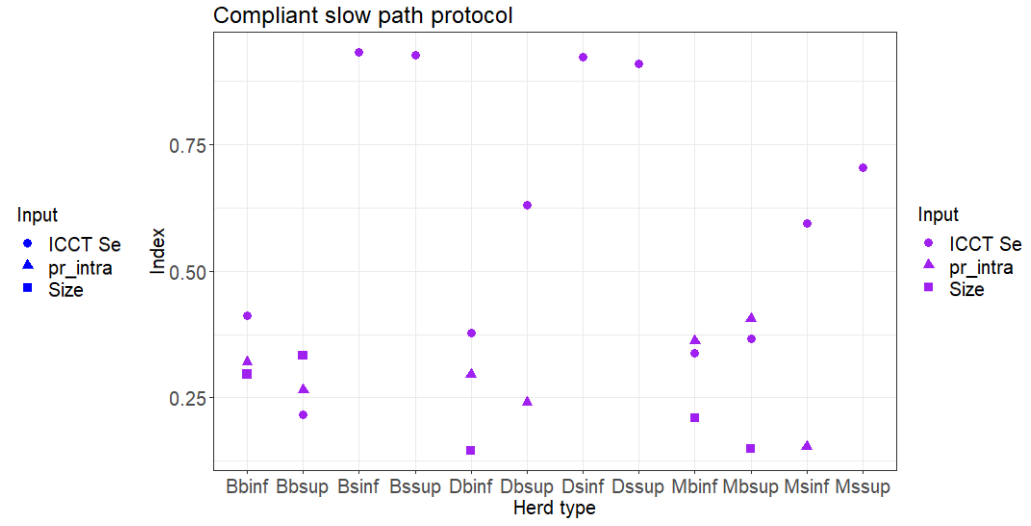

Legend: Bbinf: big beef farm with a turnover of less than $40 \%$, Bbsup: big beef farm with a turnover of greater than $40 \%$, Bsinf: small beef farm with a turnover of less than $40 \%$, Bssup: small beef farm with a turnover of greater than $40 \%$, Dbinf: big dairy farm with a turnover of less than $40 \%$, Dbsup: big dairy farm with a turnover of greater than $40 \%$, Dsinf: small dairy farm with a turnover of less than $40 \%$, Dssup: small dairy farm with a turnover of greater than $40 \%$, Mbinf: big mixed farm with a turnover of less than $40 \%$, Mbsup: big mixed farm with a turnover of greater than $40 \%$, Msinf: small mixed farm with a turnover of less than $40 \%$, Mssup: small mixed farm with a turnover of greater than $40 \%$. ICCT Se: ICCT sensitivity, pr_intra: within-herd prevalence of bTB, Size: herd size. 


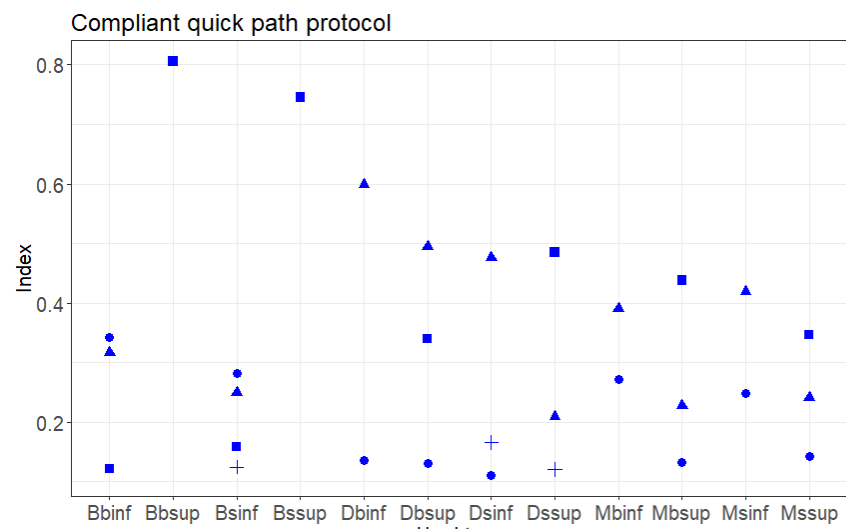

Input

- Compensation costs

- ICCT Sp

ICCT/slaughter interval

+ Size

Bbinf Bbsup Bsinf Bssup Dbinf Dbsup Dsinf Dssup Mbinf Mbsup Msinf Mssup
Herd type

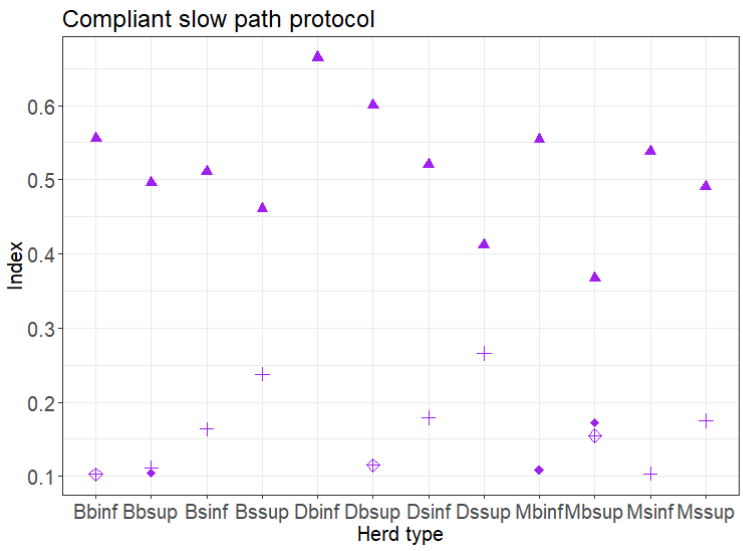

Input

- ICCT Sp

$\oplus$ IFN results/slaughter interval

+ Size

- slaughter IFNpos/slaughter interval

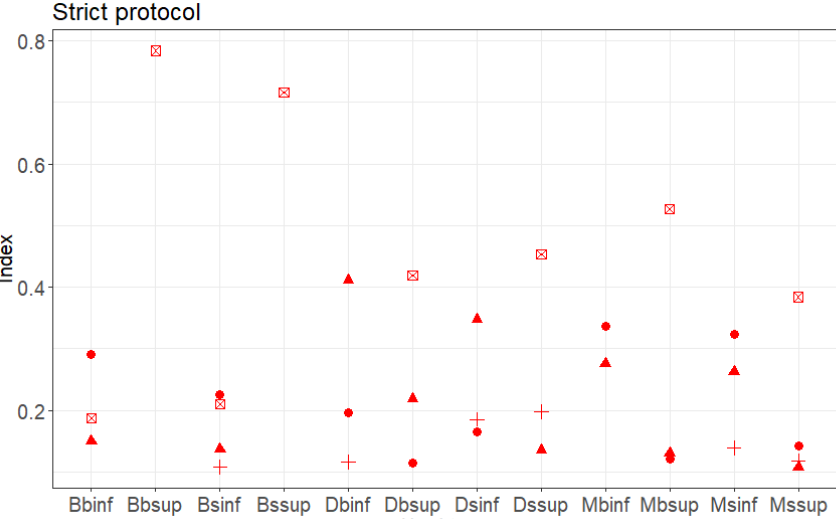

Input

- Compensation costs

ICCT Sp
ICCT $/ 2^{\text {nd }}$ ICCT interval

+ Size

Legend: Bbinf: big beef farm with a turnover of less than $40 \%$, Bbsup: big beef farm with a turnover of greater than $40 \%$, Bsinf: small beef farm with a turnover of less than $40 \%$, Bssup: small beef farm with a turnover of greater than $40 \%$, Dbinf: big dairy farm with a turnover of less than $40 \%$, Dbsup: big dairy farm with a turnover of greater than 40\%, Dsinf: small dairy farm with a turnover of less than $40 \%$, Dssup: small dairy farm with a turnover of greater than $40 \%$, Mbinf: big mixed farm with a turnover of less than $40 \%$, Mbsup: big mixed farm with a turnover of greater than $40 \%$, Msinf: small mixed farm with a turnover of less than $40 \%$, Mssup: small mixed farm with a turnover of greater than $40 \%$. Compensation costs: compensation to framers for the slaughtered animals paid by the State; ICCT Sp: specificity of ICCT; Size: herd size; ICCT/slaughter interval: interval between the first ICCT and the slaughter of non-negative animals; slaughter IFNpos/slaughter interval: interval between the slaughter of the first ICCT non-negative and IFN positive animals and the slaughter of the other first ICCT non-negative animals in the same herd; IFN result/slaughter interval: interval between results of the IFN test and the slaughter of IFN positive animals; ICCT/recontrol interval: interval between the first ICCT results and herd re-control by ICCT. 